\title{
Automatic Search for the Linear (Hull) Characteristics of ARX Ciphers: Applied to SPECK, SPARX, Chaskey, and CHAM-64
}

\author{
Mingjiang Huang $\mathbb{D}^{1,2}$ and Liming Wang $\mathbb{D}^{1}$ \\ ${ }^{1}$ State Key Laboratory of Information Security, Institute of Information Engineering, Chinese Academy of Sciences, Beijing, China \\ ${ }^{2}$ School of Cyber Security, University of Chinese Academy of Sciences, Beijing, China \\ Correspondence should be addressed to Liming Wang; wangliming@iie.ac.cn
}

Received 9 May 2019; Revised 30 August 2019; Accepted 13 November 2019; Published 21 January 2020

Academic Editor: David Megias

Copyright (c 2020 Mingjiang Huang and Liming Wang. This is an open access article distributed under the Creative Commons Attribution License, which permits unrestricted use, distribution, and reproduction in any medium, provided the original work is properly cited.

\begin{abstract}
Linear cryptanalysis is an important evaluation method for cryptographic primitives against key recovery attack. In this paper, we revisit the Walsh transformation for linear correlation calculation of modular addition, and an efficient algorithm is proposed to construct the input-output mask space of specified correlation weight. By filtering out the impossible large correlation weights in the first round, the search space of the first round can be substantially reduced. We introduce a concept of combinational linear approximation table (cLAT) for modular addition with two inputs. When one input mask is fixed, another input mask and the output mask can be obtained by the Splitting-Lookup-Recombination approach. We first split the $n$-bit fixed input mask into several subvectors and then find the corresponding bits of other masks, and in the recombination phase, pruning conditions can be used. By this approach, a large number of search branches in the middle rounds can be pruned. With the combination of the optimization strategies and the branch-and-bound search algorithm, we can improve the search efficiency for linear characteristics on ARX ciphers. The linear hulls for SPECK32/48/64 with a higher average linear potential (ALP) than existing results have been obtained. For SPARX variants, an 11-round linear trail and a 10-round linear hull have been found for SPARX-64 and a 10-round linear trail and a 9-round linear hull are obtained for SPARX-128. For Chaskey, a 5-round linear trail with a correlation of $2^{-61}$ has been obtained. For CHAM-64, 34/35-round optimal linear characteristics with a correlation of $2^{-31} / 2^{-33}$ are found.
\end{abstract}

\section{Introduction}

The three components: modular addition, rotation, and XOR, constitute the basic operations in ARX cryptographic primitives [1]. In ARX ciphers, modular additions provide nonlinearity diffusion with efficient software implementation and low dependencies on computing resources. Compared with S-box-based ciphers, ARX ciphers do not need to store S-box in advance, which can reduce the occupation of storage resources, especially in resource-constrained devices. In addition, ARX ciphers do not need to query S-boxes in the encryption and decryption process, which can reduce a lot of query operations. Therefore, ARX construction is preferred by many designers of lightweight ciphers. At present, there are many primitives used in this construction, such as HIGHT [2], SPECK [3], LEA [4], Chaskey [5], SPARX [6], and CHAM [7].
Until now, cryptanalysis on ARX ciphers is still not well understood as S-box-based ciphers, and the security analysis on them are relatively lagging behind [8]. Linear cryptanalysis is very important for evaluating the security margin of symmetric cryptographic primitives $[9,10]$. The linear approximation tables of S-box-based ciphers mostly can be constructed and stored directly; however, the full linear approximation table of modular addition will be too large to store when the word length of modular addition is large.

For linear cryptanalysis of ARX ciphers, one crucial step is to calculate the linear correlation of modular addition. In [11-14], the linear properties of modular addition have been carefully studied. In [13], a method to calculate the linear correlation of modular addition recursively was proposed, but the calculation process that was based on state transition in bit level leads to high complexity. Based on this method, 
only the optimal linear characteristics for the variants of SPECK32 [15] and SPECK32/48 [16] were found.

In 2013, Schulte-Geers used CCZ equivalence to improve the explicit formula for the calculation of linear correlation of modular addition [17]. Based on the improved formula and SAT solver model, Liu et al. obtained better linear characteristics for SPECK [18], the optimal linear trails for SPECK32/48/64 with correlation close to the security boundary $\left(2^{-n / 2}\right)$ were obtained, and the 9/10-round linear hull with a potential of $2^{-29.1} / 2^{-32.1}$ for SPECK32 was obtained.

According to the position of the starting round of the search algorithm, there are currently 3 types of automatic search technologies for linear/differential cryptanalysis on ARX primitives. They are bottom-up techniques [15], topdown techniques [19-21], and the method of extending from the middle to the ends [22]. In these methods, the linear correlations are directly calculated based on the inputoutput masks or by looking up the precomputed partial linear approximation table (pLAT) [23]. For addition modulo $2^{n}$ with two inputs, the correlations need to be calculated based on the known input-output masks. However, in the search process for linear characteristics of ARX ciphers, due to the existence of three-forked branches, in most case, for the input-output masks $((v, w) \longrightarrow u)$ of modular addition, only one input mask $v$ is determined, and another input mask $w$ and the output mask $u$ are unknown. Although all $2^{2 n}$ space of $(w, u)$ can be traversed in a trivial way, it is very time-consuming.

High efficiency query operations can be achieved by constructing a linear approximation table of reasonable storage size. The pLAT can store the input-output masks whose linear correlation is greater than a certain threshold [15]. When the branches cannot be queried in pLAT and that need to be calculated by the input-output masks, the calculation process will lead to a significant reduction in search efficiency. Although the heuristic method can speed up the search, it cannot guarantee the results will be the best [24].

Therefore, constructing a search model based on the precise correlation calculation formula and realizing an efficient search for linear characteristics on ARX ciphers are still a study worth working on. The motivation of this paper is to investigate how to speed up the search algorithm in order to realize the search for linear (hull) characteristics on typical ARX ciphers.

1.1. Our Contributions. In this paper, we first revisit the linear correlation calculation of modular addition and introduce an algorithm to construct the input-output masks of specific correlation weight. Then, we propose a novel concept of combinational linear approximation table (cLAT) and introduce an algorithm to generate the lookup tables. Combining with these two optimization algorithms, we propose an automatic algorithm to search for the optimal linear characteristics on ARX ciphers. In the first round, we can exclude the search space of the nonoptimal linear trails by increasing the correlation weight of each modular addition monotonically. In the middle rounds, the undetermined masks and the corresponding correlation weight of each modular addition can be obtained by querying the cLAT, and a large number of nonoptimal branches can be filtered out during the recombination phase. Also, the algorithm can be appropriately modified for the heuristic search.

As applications, for SPECK32/48/64, the 9/11/14-round linear hulls are obtained. For SPARX-64, the 11-round linear trail with a correlation of $2^{-28}$ and a 10 -round linear hull with an ALP of $2^{-40.92}$ are found. For SPARX-128, we can experimentally get the optimal linear trails of the first eight rounds, and we get a 10 -round linear trail with a correlation of $2^{-23}$. For Chaskey, the linear characteristics covering more rounds are updated, and a 5-round linear trail with a correlation of $2^{-61}$ is found. For CHAM-64, we find a new 34round optimal linear trail with a correlation of $2^{-31}$. A summary table is shown in Table 1.

1.2. Roadmap. This paper is organized as follows. We first present some preliminaries used in this paper in Section 2. In Section 3, we introduce the algorithm for constructing the space of input-output mask tuples, the algorithm for constructing CLAT, and the improved automatic search algorithm for linear cryptanalysis on ARX ciphers. In Section 4, we apply the new tool to several typical ARX ciphers. Finally, we conclude our work in Section 5.

\section{Preliminaries}

2.1. Notation. For addition modulo $2^{n}$, i.e., $x \boxplus y=z$, we use the symbols « and to indicate rotation to the left and right and « and $\gg$ to indicate the left and right shift operation, respectively. The binary operator symbols $\oplus, \mathrm{V}$, $\wedge$, $\|$, and $\neg$ represent XOR, OR, AND, concatenation, and bitwise NOT, respectively. For a vector $x, w t(x)$ represents its Hamming weight and $x_{i}$ is the $i^{\text {th }}$ bit of it. 0 is a zero vector.

2.2. Linear Correlation Calculation for Modular Addition. Let $\mathbb{F}_{2}^{n}$ be the $n$ dimensional vector space over binary field $\mathbb{F}_{2}=\{0,1\}$; for Boolean function $f: \mathbb{F}_{2}^{n} \longrightarrow \mathbb{F}_{2}$ and $h: \mathbb{F}_{2}^{n} \longrightarrow \mathbb{F}_{2}, x \in \mathbb{F}_{2}^{n}$, the linear correlation between $f$ and $h$ can be denoted by

$$
\operatorname{Cor}(f, h)=2 \times \frac{\#\{x \mid f(x) \oplus h(x)=0\}}{2^{n}}-1 .
$$

For modular addition $x \boxplus y=z$, let $(v, w)$ be the input masks, $u$ be the output mask, and $\cdot$ be the standard inner product. According to the definition of linear correlation, when $(v \cdot x \boxplus w \cdot y) \oplus u \cdot z=0$, the linear approximation probability is defined as

$$
\operatorname{Pr}(u, v, w)=2^{-3 n} \times \#\{(x, y, z) \mid(v \cdot x \boxplus w \cdot y) \oplus u \cdot z=0\} .
$$

Let $\mu(t)=(-1)^{t}$, then the linear correlation of modular addition can be denoted by Walsh transformation, and thus, 
TABLE 1: Summary of the linear characteristics on SPECK, SPARX, Chaskey, and CHAM-64, where "s," "m," "h," and "d" represent seconds, minutes, hours, and days, respectively.

\begin{tabular}{lcccccc}
\hline Variants & Round & Cor & $T_{\text {Cor }}$ & ALP & $T_{\text {ALP }}$ & Reference \\
\hline \multirow{4}{*}{ SPECK32 } & 9 & $2^{-14}$ & N/A & $2^{-29.1}$ & N/A & {$[18]$} \\
& 9 & $2^{-14}$ & N/A & $2^{-28}$ & N/A & {$[16]$} \\
& 9 & $2^{-14}$ & $9 \mathrm{~s}$ & $2^{-27.78}$ & $25 \mathrm{~s}$ & This paper \\
\hline \multirow{3}{*}{ SPECK48 } & 10 & $2^{-22}$ & N/A & $2^{-44}$ & N/A & {$[18]$} \\
& 10 & $2^{-22}$ & N/A & $2^{-44}$ & N/A & {$[16]$} \\
& 10 & $2^{-22}$ & $3.2 \mathrm{~h}$ & $2^{-43.64}$ & $157.3 \mathrm{~h}$ & This paper \\
\hline \multirow{5}{*}{ SPECK64 } & 13 & $2^{-30}$ & N/A & $2^{-60}$ & N/A & {$[18]$} \\
& 13 & $2^{-30}$ & N/A & $2^{-60}$ & N/A & {$[16]$} \\
& 13 & $2^{-30}$ & $8.6 \mathrm{~h}$ & $2^{-55.29}$ & $7.3 \mathrm{~h}$ & This paper \\
\multirow{2}{*}{ SPARX-64 } & 14 & $2^{-33}$ & $25.6 \mathrm{~h}$ & $2^{-61.24}$ & $5.8 \mathrm{~h}$ & This paper \\
& 10 & $2^{-22}$ & $3 \mathrm{~d}$ & $2^{-40.92}$ & $1 \mathrm{~h}$ & This paper \\
\multirow{2}{*}{ SPARX-128 } & 11 & $2^{-28}$ & $5 \mathrm{~m}$ & $2^{-56}$ & - & This paper \\
& 9 & $2^{-18}$ & $27 \mathrm{~m}$ & $2^{-35.22}$ & $6 \mathrm{~h}$ & This paper \\
& 10 & $2^{-23}$ & $4.4 \mathrm{~d}$ & $2^{-46}$ & - & This paper \\
\multirow{3}{*}{ Chaskey } & 3 & $2^{-9}$ & N/A & $2^{-18}$ & N/A & {$[18]$} \\
& 4 & $2^{-29}$ & $15.7 \mathrm{~m}$ & $2^{-58}$ & - & This paper \\
& 5 & $2^{-61}$ & $6.6 \mathrm{~h}$ & $2^{-122}$ & - & This paper \\
\hline \multirow{2}{*}{ CHAM-64 } & 34 & $2^{-31}$ & N/A & $2^{-62}$ & N/A & {$[7]$} \\
& 34 & $2^{-31}$ & $1.1 \mathrm{~d}$ & $2^{-62}$ & - & This paper \\
& 35 & $2^{-33}$ & $4.8 \mathrm{~d}$ & - & - & This paper \\
\hline
\end{tabular}

$$
\operatorname{Cor}(u, v, w)=2^{-3 n} \times \sum_{x, y, z \in \mathbb{F}_{2}^{n}} \mu((v \cdot x \boxplus w \cdot y) \oplus u \cdot z) .
$$

Let $\operatorname{Pr}(u, v, w)=(1 / 2)+\varepsilon$, where $\varepsilon$ is the bias. When $(v \cdot x \boxplus w \cdot y) \oplus u \cdot z=1$, the linear approximation probability is $\overline{\operatorname{Pr}}(u, v, w)=(1 / 2)-\varepsilon$. The linear correlation can be denoted by

$$
\operatorname{Cor}(u, v, w)=\operatorname{Pr}(u, v, w)-\overline{\operatorname{Pr}}(u, v, w)=2 \operatorname{Pr}(u, v, w)-1 .
$$

We call $\mathrm{Cw}(u, v, w)=-\log _{2} \operatorname{Cor}(u, v, w)$ as the correlation weight, and the linear square correlation can be denoted by

$$
\operatorname{LSC}(u, v, w)=\operatorname{Cor}(u, v, w)^{2}=2^{-2 \times \operatorname{Cw}(u, v, w)} .
$$

For addition $x \boxplus y$ modulo $2^{n}$, it can be rewritten as $x \boxplus y=x \oplus y \oplus \operatorname{carry}(x, y), \quad$ in which $\operatorname{carry}(x, y)_{i+1}=$ $\operatorname{carry}(x, y)_{i} \oplus x_{i} \oplus y_{i}$ and $\operatorname{carry}(x, y)_{0}=0$ for $0 \leq i \leq n-1$. The first-order approximation is carry $(x, y)=(x \wedge y) \ll 1$. If all carry $(x, y)_{j}=0$ for $j \leq i$, and $0 \leq i \leq n-1$, the highorder approximation is

$$
\begin{aligned}
\operatorname{carry}(x, y)_{i+1}= & \frac{1}{2} \mid(-1)^{x_{i}}+(-1)^{y_{i}}+\operatorname{carry}(x, y)_{i} \\
& -(-1)^{x_{i}+y_{i}} \operatorname{carry}(x, y)_{i} \mid .
\end{aligned}
$$

In [13], Wallén introduced the theorem to calculate the linear correlation by analyzing the carry high-order approximation function recursively. In [12], based on the bit state transformation, the formula to calculate the correlation was given by the following theorem.
Theorem 1 (See [12]). For addition modulo $2^{n}$, let $v$ and $w$ be the input masks and $u$ be the output mask. Define an auxiliary vector $d=d_{n-1} \cdots d_{0}$, and each $d_{i}=u_{i}\left\|v_{i}\right\| w_{i} \in \mathbb{F}_{2}^{3}$ is an octal word, $0 \leq i \leq n-1$. Then, the linear correlation can be denoted by

$$
\operatorname{Cor}(u, v, w)=L A_{d_{n-1}} A_{d_{n-2}} \cdots A_{d_{1}} A_{d_{0}} C,
$$

where the row vector $L=(10)$, the column vector $C=\left(\begin{array}{ll}1 & 1\end{array}\right)^{T}$, and each $2 \times 2$ matrix $A_{d_{i}}$ is defined by

$$
\begin{aligned}
& A_{0}=\frac{1}{2}\left(\begin{array}{ll}
2 & 0 \\
0 & 1
\end{array}\right), A_{1}=A_{2}=-A_{4}=\frac{1}{2}\left(\begin{array}{ll}
0 & 0 \\
1 & 0
\end{array}\right), \\
& A_{7}=\frac{1}{2}\left(\begin{array}{ll}
0 & 2 \\
1 & 0
\end{array}\right),-A_{3}=A_{5}=A_{6}=\frac{1}{2}\left(\begin{array}{ll}
0 & 0 \\
0 & 1
\end{array}\right) .
\end{aligned}
$$

In [17], Schulte-Geers extended Theorem 1 and derived a fully explicit formula for the linear correlation calculation, given by Theorem 2 .

Theorem 2 (See [17]). For addition modulo $2^{n}$ with inputoutput mask tuple $(u, v, w)$, a vectorial Boolean function $M$ : $\mathbb{F}_{2}^{n} \longrightarrow \mathbb{F}_{2}^{n}$ denotes the partial sum mapping:

$$
\begin{aligned}
x & =\left(x_{0}, x_{1}, \cdots, x_{n-1}\right) \longrightarrow M(x) \\
& =\left(0, x_{0}, x_{0} \oplus x_{1}, \cdots, x_{0} \oplus x_{1} \oplus \cdots x_{n-2}\right) .
\end{aligned}
$$

Let $\quad z:=M^{T}(u \oplus v \oplus w)=\left(0, x_{n-1}, x_{n-1} \oplus x_{n-2}, \cdots, x_{n-1}\right.$ $\left.\oplus x_{n-2} \oplus \cdots x_{1}\right)$, then the linear correlation can be denoted by

$$
\operatorname{Cor}(u, v, w)=1_{\{u \oplus v \leq z\}} 1_{\{u \oplus w \leq z\}}(-1)^{v \cdot w} 2^{-w t(z)},
$$

where $1_{G_{f}}$ is an indicator function for graph $G_{f}:=$ $\left\{(x, f(x))^{f} \mid x \in \mathbb{F}_{2}^{n}\right\}$; for $n$-bit vectors $a$ and $b, a \preceq b$ represents $a_{i} \leq b_{i}$ for $0 \leq i \leq n-1$.

In iterative ciphers, the correlation of a single $r$-round linear trail is the product of the correlations of each round [25]. Assuming that there are $N_{A}$ additions modulo $2^{n}$ with two inputs in $i^{\text {th }}$ round, $\Gamma_{\text {in }}$ and $\Gamma_{\text {out }}$ are the input and output masks of the $r$-round linear trail, and the correlation of it can be denoted by

$$
\operatorname{Cor}\left(\Gamma_{\text {in }}, \Gamma_{\text {out }}\right)=\prod_{i=1}^{r} \prod_{j=1}^{N_{A}} \operatorname{Cor}\left(u_{i, j}, v_{i, j}, w_{i, j}\right) .
$$

The linear approximation of a linear hull represents the potential of all linear trails with same input-output masks [26]. The averaged linear potential (ALP) can be counted by the following formula:

$$
\operatorname{ALP}\left(\Gamma_{\text {in }}, \Gamma_{\text {out }}\right)=\frac{1}{|K|} \sum_{k \in K} \operatorname{Cor}\left(\Gamma_{\text {in }}, \Gamma_{\text {out }}\right)^{2} .
$$

Assuming that the key $k$ is selected uniformly from the key space $K$, the statistics of ALP can be formulated as (13), where $T[C w]$ is the number of trails with correlation weight of $\mathrm{Cw}$. Let $C_{\min }$ be the correlation weight of the linear trail 
whose input-output masks are chosen as the fixed inputoutput masks of the linear hull. $C_{\max }$ is the upper bound to be searched, which should be chosen by the trade-off between the search time and the accuracy of ALP:

$$
\operatorname{ALP}\left(\Gamma_{\text {in }}, \Gamma_{\text {out }}\right)=\sum_{C w=C_{\min }}^{C_{\max }} 2^{-2 C w} \times T[C w] .
$$

2.3. Linear Properties of SPECK, SPARX, Chaskey, and CHAM. The SPECK family ciphers were designed by NSA in 2013 [3]. The SPARX family ciphers were introduced by Dinu et al. at ASIACRYPT'16 [6]. In SPARX, the nonlinear ARX-box (SPECKEY) is obtained by modifying the round function of SPECK32. The linear mask propagation properties of the round function in SPECK and SPECKEY are shown in Figure 1. The rotation parameters $\left(r_{a}, r_{b}\right)=(7,2)$ for SPECK32, while $\left(r_{a}, r_{b}\right)=(8,3)$ for other variants.

If the input-output masks $(u[i], v[i], w[i])$ and $(u[i+1], v[i+1], w[i+1])$ of the modular additions in the two consecutive rounds of SPECK are known, the input and output masks of these two rounds can be denoted by Property 1.

Property 1. If $(u[i], v[i], w[i])$ and $(u[i+1], v[i+1], w[i+$ 1]) are given, then $\Gamma X[i]=v[i] \lll r_{a}, \Gamma X[i+1]=v[i+$ $1] \lll r_{a}, \Gamma Y[i+1]=\left(v[i+1] \lll r_{a}\right) \oplus u[i], \Gamma Y[i]=(\Gamma Y[i+$ 1] $\left.r_{b}\right) \oplus w[i], \quad \Gamma Y[i+2]=(\Gamma Y[i+1] \oplus w[i+1]) \lll r_{b}$, and $\Gamma X[i+2]=\Gamma Y[i+1] \oplus u[i+1]$.

The linear layer functions $\mathcal{L} / \mathcal{L}^{\prime}$ [6] for SPARX-64 and SPARX-128 are shown in Figure 2. Due to the existence of the three-forked branches, the masks of the linear transformation layer have the following properties.

Property 2. For SPARX-64, if the masks are transformed by the linear layer function $\mathcal{L}$, let $c=\Gamma X_{2} \oplus \Gamma X_{3}, d=c \gg 8$, then $\Gamma X_{0}^{\prime}=\Gamma X_{2}, \quad \Gamma X_{1}^{\prime}=\Gamma X_{3}, \quad \Gamma X_{2}^{\prime}=\Gamma X_{0} \oplus d \oplus \Gamma X_{2}$, and $\Gamma X_{3}^{\prime}=\Gamma X_{1} \oplus d \oplus \Gamma X_{3}$.

Property 3. For SPARX-128, if the masks are transformed by the linear layer function $\mathcal{L}^{\prime}$, let $e=\Gamma X_{4} \oplus \Gamma X_{5} \oplus$ $\Gamma X_{6} \oplus \Gamma X_{7}, f=e \ggg 8$, then $\Gamma X_{0}^{\prime}=\Gamma X_{4}, \Gamma X_{1}^{\prime}=\Gamma X_{5}, \Gamma X_{2}^{\prime}=$ $\Gamma X_{6}, \Gamma X_{3}^{\prime}=\Gamma X_{7}, \quad \Gamma X_{4}^{\prime}=\Gamma X_{0} \oplus f \oplus \Gamma X_{6}, \quad \Gamma X_{5}^{\prime}=\Gamma X_{1} \oplus f \oplus$ $\Gamma X_{5}, \Gamma X_{6}^{\prime}=\Gamma X_{2} \oplus f \oplus \Gamma X_{4}$, and $\Gamma X_{7}^{\prime}=\Gamma X_{3} \oplus f \oplus \Gamma X_{7}$.

Chaskey is a MAC algorithm introduced by Mouha et al. at $\mathrm{SAC}^{\prime} 14$ [5], and an enhanced variant was proposed in 2015 [27], which increases the number of permutation rounds from 8 to 12 . The round function of the permutation $\left(v 0^{\prime}, v 1^{\prime}, v 2^{\prime}, v 3^{\prime}\right)=\pi(v 0, v 1, v 2, v 3)$ is shown in Figure 3. The 4 modular additions are labeled by $A 0, A 1, A 2$, and $A 3$, respectively. The input mask $(a, b, c, d)$ and the output mask $\left(a^{\prime}, b^{\prime}, c^{\prime}, d^{\prime}\right)$ of the first round can be denoted by Property 4 .

Property 4. For the permutation of Chaskey, if the inputoutput masks of each modular addition in the first round are $(u[i], v[i], w[i]), \quad 0 \leq i \leq 3$, the corresponding correlation weight of each modular addition is $c_{0}, c_{1}, c_{2}, c_{3}$, respectively. Hence, in the first round, $a=v[0], b=w[0] \oplus((u[0] \oplus$ $(v[3] \ggg 16))) \ggg 5, c=v[1], d=w[1] \oplus(u[1] \oplus v[2]) \ggg 8$,
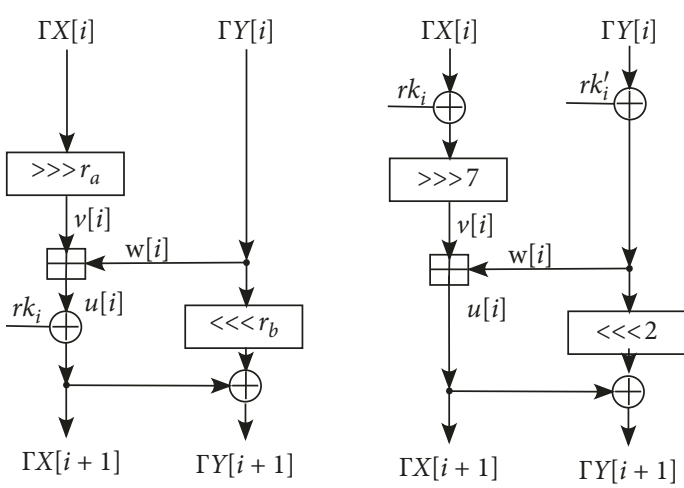

FIGURE 1: Linear mask propagation properties of SPECK and SPECKEY.

$a^{\prime}=u[3] \oplus(u[1] \oplus v[2] \oplus w[3]) \lll 13, \quad b^{\prime}=(u[0] \oplus w[2] \oplus$ $w[3] \ggg 16) \lll 7, \quad c^{\prime}=u[2] \oplus(w[2] \oplus(u[0] \oplus v[3]) \ggg 16)$ $\lll 7$, and $d^{\prime}=(u[1] \oplus v[2] \oplus w[3]) \lll 13$. The corresponding correlation weight of the round function is $C w=\sum_{i=0}^{3} c_{i}$.

CHAM is a family of lightweight block ciphers that was proposed by Koo et al. at ICISC'17, which blends the good designs of SIMON and SPECK [7]. 3 variants of CHAM have two kinds of block size, i.e., CHAM-64 and CHAM-128. The linear mask propagation for the 4 consecutive rounds of CHAM is shown in Figure 4. If the input-output mask tuples of each modular addition of the first 4 rounds are given, the input and output masks of the first 4 rounds can be deduced by Property 5 .

Property 5. For CHAM, if the input-output mask tuples $(u[i], v[i], w[i])$ of each modular addition of the first 4 rounds are given, $1 \leq i \leq 4$, the input and output masks of the first 4 rounds can be deduced as follows. $\Gamma X_{0}^{0}=v[1]$, $\Gamma X_{1}^{0}=(w[1] \lll 1) \oplus v[2], \Gamma X_{2}^{0}=(w[2] \lll 8) \oplus v[3], \Gamma X_{3}^{0}=$ $(w[3] \lll 1) \oplus v[4] ; \quad \Gamma X_{0}^{1}=v[2], \quad \Gamma X_{1}^{1}=\Gamma X_{2}^{0}, \quad \Gamma X_{2}^{1}=\Gamma X_{3}^{0}$, $\Gamma X_{3}^{1}=u[1] \lll 8 ; \Gamma X_{0}^{2}=v[3], \Gamma X_{1}^{2}=\Gamma X_{2}^{1}, \Gamma X_{2}^{2}=\Gamma X_{3}^{1}, \Gamma X_{3}^{1}=$ $u[2] \lll 1 ; \Gamma X_{0}^{3}=v[4], \Gamma X_{1}^{3}=\Gamma X_{2}^{2}, \Gamma X_{2}^{3}=\Gamma X_{3}^{2}, \Gamma X_{3}^{3}=u[3]$ $\lll 8 ; \Gamma X_{0}^{4}=(w[4] \lll 8) \oplus \Gamma X_{1}^{3}, \Gamma X_{1}^{4}=\Gamma X_{2}^{3}, \Gamma X_{2}^{4}=\Gamma X_{3}^{3}, \Gamma X_{3}^{4}$ $=u[4] \lll 1$.

\section{Automatic Search for the Linear Characteristics on ARX Ciphers}

3.1. Input-Output Masks of Specific Correlation Weight. The number of input-output mask tuples in the first round is closely related to the complexity of the branch-and-bound search algorithm, but traversing all possible input masks of the first round will result in high complexity. An alternative approach is to consider the possible correlation weight corresponding to the input-output masks and exclude those tuples that have a large correlation weight. However, for a fixed correlation weight, it may correspond to multiple input-output mask tuples although the correlation can be calculated by Theorem 2 when the input-output masks are fixed for a modular addition.

For addition modulo $2^{n}$, its maximum correlation weight is $n-1$, and the size of the total space $S$ of all input-output 


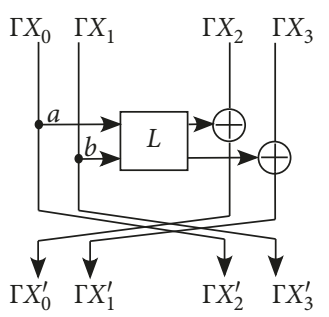

(a)

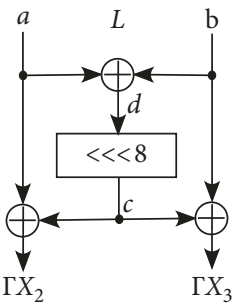

(b)

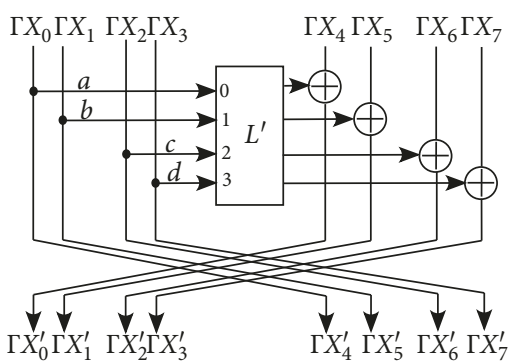

(c)

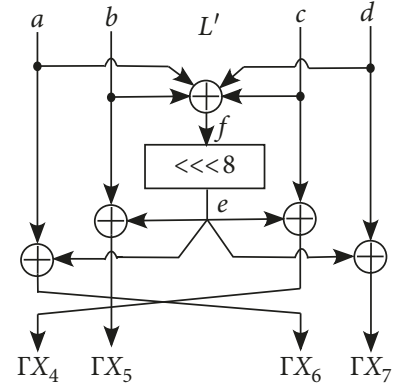

(d)

Figure 2: (a) and (b) represent the linear layer of SPARX-64. (c) and (d) represent the linear layer of SPARX-128.

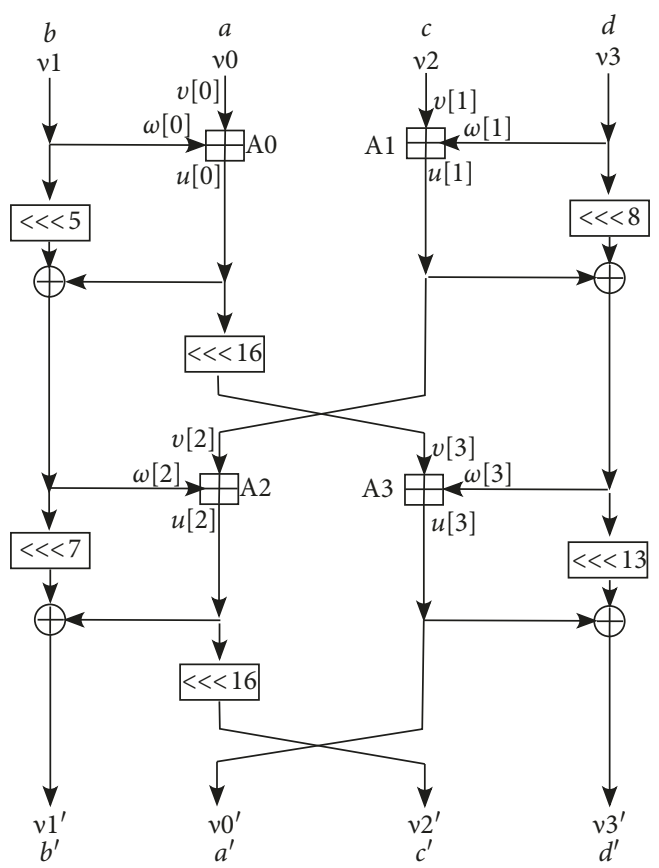

Figure 3: Linear masks in the first round of Chaskey.

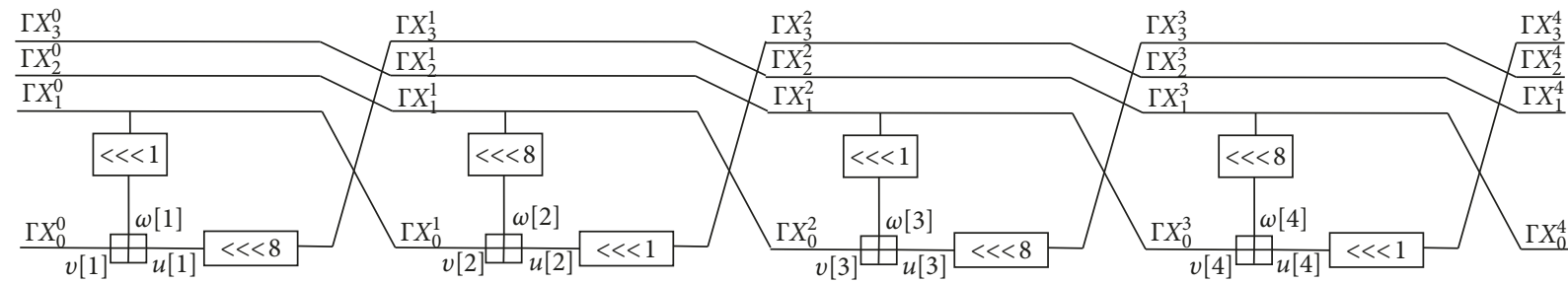

Figure 4: Linear masks in the first 4 rounds of CHAM.

mask tuples is $2^{3 n}$. We can rank the correlation weights $C w$ from 0 to $n-1$ and construct the input-output masks subspace $S_{C w}$ corresponding to correlation weight $C w$, $0 \leq C w \leq n-1$. Therefore, the total space $S$ can be divided into $n$ subspaces, i.e., $S=\cup_{C w=0}^{n-1} S_{C w}$.

Definition 1. Let $((v, w) \longrightarrow u)$ be the input-output masks for a modular addition with nonzero correlation. Let us define an octal word sequence $\Phi:=\left\{\xi_{n-1} \ldots \xi_{0}\right\}$, where $\xi_{i}=u_{i}\left\|v_{i}\right\| w_{i} \in \mathbb{F}_{2}^{3}$, for $0 \leq i \leq n-1$.

Definition 2. Let us define three sets that $\xi_{i}$ may belong to, i.e., $U_{0}=\{1,2,4,7\}, U_{1}=\{0,3,5,6\}$, and $U_{2}=\{0,7\}$.

In Theorem 2, when the correlation of a modular addition is nonzero, the value distribution of the 3 consecutive bits in $z$ and the 3 consecutive words in 
$\Phi$ has the following relationships, shown in Observation 1.

Observation 1. Let $x=u \oplus v \oplus w$ and $z_{i}=\oplus_{j=i+1}^{n-1} x_{j}$ for $0 \leq i \leq n-2, z_{n-1}=0$; hence, $z_{i}=z_{i+1} \oplus x_{i+1}$. For $z \in \mathbb{F}_{2}^{n}$, assuming when $z_{j}=0$ for $n-1 \geq j>i+1$ or $z_{i+2}=0$, they should have $u_{i} \oplus v_{i} \preceq z_{i}, u_{i} \oplus w_{i} \preceq z_{i}$ on bit level, and it is equivalent to $\quad u_{i-1} \oplus v_{i-1} \preceq x_{i+1} \oplus x_{i} \quad$ and $u_{i-1} \oplus w_{i-1} \preceq x_{i+1} \oplus x_{i}$. Since $x_{i}=1$ when $\xi_{i} \in U_{0}$ and $x_{i}=0$ when $\xi_{i} \in U_{1}$,

$$
\begin{aligned}
& \text { if }\left(z_{i+1}, z_{i}, z_{i-1}\right)=(0,0,0), \quad \text { then } \xi_{i+1}=0, \xi_{i}=0, \xi_{i-1}=0 \text {; } \\
& \text { if }\left(z_{i+1}, z_{i}, z_{i-1}\right)=(0,0,1) \text {, then } \xi_{i+1}=0, \xi_{i}=0, \xi_{i-1}=7 \text {; } \\
& \text { if }\left(z_{i+1}, z_{i}, z_{i-1}\right)=(0,1,0) \text {, then } \xi_{i+1}=0, \xi_{i}=7, \xi_{i-1} \in U_{0} \text {; } \\
& \text { if }\left(z_{i+1}, z_{i}, z_{i-1}\right)=(0,1,1), \quad \text { then } \xi_{i+1}=0, \xi_{i}=7, \xi_{i-1} \in U_{1} \text {; } \\
& \text { if }\left(z_{i+1}, z_{i}, z_{i-1}\right)=(1,0,0) \text {, then } \xi_{i+1}=7, \xi_{i}=U_{0}, \xi_{i-1}=0 \text {; } \\
& \text { if }\left(z_{i+1}, z_{i}, z_{i-1}\right)=(1,0,1) \text {, then } \xi_{i+1}=7, \xi_{i}=U_{0}, \xi_{i-1}=7 \text {; } \\
& \text { if }\left(z_{i+1}, z_{i}, z_{i-1}\right)=(1,1,0) \text {, then } \xi_{i+1}=7, \xi_{i}=U_{1}, \xi_{i-1} \in U_{0} \text {; } \\
& \text { if }\left(z_{i+1}, z_{i}, z_{i-1}\right)=(1,1,1) \text {, then } \xi_{i+1}=7, \xi_{i}=U_{1}, \xi_{i-1} \in U_{1} \text {; }
\end{aligned}
$$

Hence, the value of $\xi_{i}=u_{i}\left\|v_{i}\right\| w_{i}$ depends on whether the bit positions of $z_{i+1}$ and $z_{i}$ are active. The last significant bits $\left(u_{0}, v_{0}, w_{0}\right)$ of the input-output masks construct the value of $\xi_{0}$, which is only related to the Hamming weight of $z_{0}=\oplus_{j=1}^{n-1} x_{j}$, i.e., $u_{0} \oplus v_{0} \preceq w t\left(z_{0}\right)$ and $u_{0} \oplus w_{0} \preceq w t\left(z_{0}\right)$. Therefore, if we get the Hamming weight distribution of $z$, from the LSB to MSB direction, as $z_{0}$ is determined, $\xi_{0}$ can be obtained. Next, $x_{1}=z_{1} \oplus z_{0}$ is determined, and $u_{1} \oplus v_{1} \preceq w t\left(z_{1}\right)$ and $u_{1} \oplus w_{1} \preceq w t\left(z_{1}\right)$ should be satisfied; hence, the possible values of $\xi_{1}$ can be obtained. Recursively, all $\xi_{i}$ values can be constructed as an octal word sequence from the LSB to MSB direction to subject to the above observation. Hence, the tuples of $(u, v, w)$ can be generated from the elements in $\Phi$. The process to construct the subspace $S_{C w}$ is shown in Algorithm 1, marked as Const $\left(S_{C w}\right)$.

3.2. Combinational Linear Approximation Table. For addition modulo $2^{n}$, the full LAT requires a storage size of $2^{3 n}$; when $n$ is too large, it will be very difficult to store. To facilitate the storage, an intuitive approach is to store only a part of the full LAT. For a $n$-bit vector, we can split it into $t$ subvectors of $m$ bits, where $n=m t$. When each $m$-bit subvector is determined, the $n$-bit vector can be obtained by concatenating. This idea gives birth to the concept of combinatorial LAT (cLAT).

Property 6. For $u \oplus v \preceq z$ and $u \oplus w \preceq z$, they are equivalent to $(u \oplus v) \wedge(\neg((u \oplus v) \wedge \vec{z}))=\overrightarrow{0}$ and $(u \oplus w) \wedge$ $(\neg((u \oplus w) \wedge \vec{z}))=\overrightarrow{0}$.

Corollary 1. Let $u, v, w \in \mathbb{F}_{2}^{n}$ be the input-output masks of the modular addition with nonzero correlation, and let $A=u \oplus v, \quad B=u \oplus w, \quad C=u \oplus v \oplus w, \quad$ and $\quad z=M^{T}(C)$.
Splitting the vectors $A=A^{t-1}\|\cdots\| A^{0}, B=B^{t-1}\|\cdots\| B^{0}, C=$ $C^{t-1}\|\cdots\| C^{0}$, and $z=z^{t-1}\|\cdots\| z^{0}$ into $t$ subvectors, respectively, $n=m t, A^{k}, B^{k}, C^{k} \in \mathbb{F}_{2}^{m}, 0 \leq k \leq t-1$. Then, the correlation weight of the modular addition can be denoted by

$$
-\log _{2} \operatorname{Cor}(u, v, w)=\sum_{k=0}^{t-1} w t\left(z^{k}\right)=\sum_{k=0}^{t-1} \sum_{j=0}^{m-1} C_{m k+j+1} \oplus z_{m k+j+1}
$$

when

$$
\begin{aligned}
& A^{k} \wedge\left(\neg\left(A^{k} \wedge z^{k}\right)\right)=0, \\
& B^{k} \wedge\left(\neg\left(B^{k} \wedge z^{k}\right)\right)=0 .
\end{aligned}
$$

Proof. $w t(z)$ is the sum of the Hamming weight of each subvector $z^{k}$, so $-\log _{2} \operatorname{Cor}(u, v, w)=\sum_{k=0}^{t-1} w t\left(z^{k}\right)$. For $C=$ $u \oplus v \oplus w$, the $i^{\text {th }}$ bit in $z$ can be denoted by $z_{i}=\oplus_{j=i+1}^{n-1}$ $C_{j}=z_{i+1} \oplus C_{i+1}$. Let $0 \leq j<i, 0 \leq i \leq n-1$, and the $j^{\text {th }}$ bit in $z^{k}$ should be $z_{j}^{k}=C_{m k+j+1} \oplus z_{m k+j+1}$. Hence, $w t\left(z^{k}\right)=\sum_{j=0}^{m-1}$ $C_{m k+j+1} \oplus z_{m k+j+1}$, when $u_{i} \oplus v_{i} \preceq \oplus_{l=i+1}^{n-1} C_{l}$ and $u_{i} \oplus w_{i} \preceq$ $\oplus_{l=i+1}^{n-1} C_{l}$ are satisfied, i.e., $A^{k} \wedge\left(\neg\left(A^{k} \wedge z^{k}\right)\right)=0$ and $B^{k} \wedge\left(\neg\left(B^{k} \wedge z^{k}\right)\right)=0$ for $0 \leq k \leq t-1$.

If the $m$-bit subvector $z^{k+1}$ adjacent to $z^{k}$ is known, $z^{k}$ can be calculated by subvector tuple $\left(u^{k}, v^{k}, w^{k}\right)$ and the lowest bit of $z^{k+1}$. We call $\left(u^{k}, v^{k}, w^{k}\right)$ as a subblock, and we call the bit $z_{(k+1) m} \in\{0,1\}$ as the connection status when used in the calculation of $z^{k}$. Splitting the $n$-bit vector $z$ into $t$ subvectors, there should have $t-1$ connection status $z_{j}$, $j \in\{(t-1) m, \cdots, 2 m, m\}$, and for the highest subvector, its connection status $b=0$. Hence, for the highest subblock $\left(u^{t-1}, v^{t-1}\right.$, and $\left.w^{t-1}\right)$, the Hamming weight of $z^{t-1}$ and the bit $z_{(t-1) m}$ can be obtained, recursively, and the Hamming weight of the remaining subvectors can also be obtained. Therefore, as connection status $b \in\{0,1\}$ and $u^{k}, v^{k}, w^{k} \in \mathbb{F}_{2}^{m}$, we can construct a $m$-bit lookup table for modular addition in advance and query the tables by indexing input-output masks and the connection status. In addition, the connection status for the next subblock can also be generated.

In the top-down search techniques for the ARX ciphers, for the modular additions in the middle rounds, in most cases, only one input mask is fixed (assuming it is $v$ ), and another input mask $w$ and the output mask $u$ are unknown. In the lookup tables, we need to lookup all valid subvectors of $(u, w)$ that correspond to nonzero correlation based on $v$. The lookup table (called as cLAT) is constructed by Algorithm 2, and it takes about 4 seconds on a $2.5 \mathrm{GHz}$ CPU to generate the table with storage size about 1.2 GByte when $m=8$.

3.3. Splitting-Lookup-Recombination. Algorithm 2 constructs a $m$-bit cLAT for the addition modulo $2^{n}$, and this section describes how to use it. When one input mask is fixed, we can get another input mask, the output mask, and the corresponding correlation weight by the SplittingLookup-Recombination approach, which contains three steps. 
Input: $C w$ and $\Lambda=\left\{\lambda_{C w}, \ldots, \lambda_{1}\right\}$. Each pattern of the Hamming weight distribution of $z$ can be calculated by the combination algorithm in [28], which is the combination pattern of $\left(\begin{array}{c}n-1 \\ C w\end{array}\right)$, where $\xi_{i}=u_{i}\left\|v_{i}\right\| w_{i}$ for $0 \leq i \leq n-1$.
LSB: $i=0 / /$ constructing the LSBs of $u, v$, and $w$.

(1) Func_LSB: $i=0$
(2) if $C w=0$ then

(3) Output the tuple of $(u, v, w)$ with $(1,1,1)$ or $(0,0,0)$;

(4) end if

(5) if $\lambda_{1} \neq 0$ then

(6) For each $\xi_{i} \in U_{2}, c=1$, and $F w=0$, call Func_Middle $(i+1, c, F w)$;

(7) else

(8) For each $\xi_{i} \in \mathbb{F}_{2}^{3}, c=2$, and $F w=1$, call Func_Middle $(i+1, c, F w)$;

(9) end if

(10) Func_Middle $(i, c, F w)$ : //constructing the middle bits of $u, v$, and $w$.

(11) if $c=C w$ then

(12) call Func_MSB $(i, c, F w)$;

(13) end if

(14) if $\lambda_{c} \neq i$ then

(15) if $F w=0$ then $/ / F w$ recorded whether the value of $\lambda_{i-1}$ is 1 or not.

(16) For each $\xi_{i}=0$ and $F w^{\prime}=0$, call Func_Middle $\left(i+1, c, F w^{\prime}\right)$;

(17) else

(18) For each $\xi_{i}=7$ and $F w^{\prime}=0$, call Func_Middle $\left(i+1, c, F w^{\prime}\right)$;

(19) end if

(20) else $/ / \lambda_{c}=i$. The value of $F w$ determines whether $\xi_{i}$ belongs to $U_{0}$ or $U_{1}$.

(21) if $F w=0$ then

(22) For each $\xi_{i} \in U_{0}$ and $F w^{\prime}=1$, call Func_Middle $\left(i+1, c+1, F w^{\prime}\right)$;

(23) else

(24) For each $\xi_{i} \in U_{1}$ and $F w^{\prime}=1$, call Func_Middle $\left(i+1, c+1, F w^{\prime}\right)$;

(25) end if

(26) end if

(27) Func_MSB $(i, c, F w)$ : //constructing the bits of $u, v, w$ with position higher than $\lambda_{C w}$.

(28) if $\lambda_{c} \neq i$ then //the value of $F w$ determines whether $\xi_{i}$ equals to 0 or 7 .

(29) if $F w=0$ then

(30) Let $\xi_{i}=0$ and $F w^{\prime}=0$, call Func_MSB $\left(i+1, c, F w^{\prime}\right)$;

(31) else

(32) Let $\xi_{i}=7$ and $F w^{\prime}=0$, call Func_MSB $\left(i+1, c, F w^{\prime}\right)$;

(33) end if

(34) else $/ / \lambda_{c}=i$.

(35) if $F w=0$ then

(36) For each $\xi_{i} \in U_{0}$ and $\xi_{i+1}=7$, output each tuple of $(u, v, w)$;

(37) else

(38)

(39) end if

For each $\xi_{i} \in U_{1}$ and $\xi_{i+1}=7$, output each tuple of $(u, v, w)$;

(40) end if

Algorithm 1: Const $\left(S_{C w}\right)$ : constructing the input-output mask tuples with linear correlation weight of $C w$ for modular addition, $0 \leq C w \leq n-1$.

3.3.1. Splitting. For addition modulo $2^{n}, n=m t$, if one of the two input masks $v$ is fixed, then split $v$ into $t m$-bit subvectors. The larger the $m$, the fewer the times to lookup cLAT and the fewer the number of bit concatenation operations, but the more space the memory takes up, and after the trade-offs, we choose $m=8$.

3.3.2. Lookup. From the MSB to the LSB direction, query the subvectors of $(u, w)$ that correspond to each subvector of $v$ and the corresponding correlation weights. For the highest $m$-bit subvector $v^{t-1}$, its connection status $b=0$, looking up cLAT to get $w^{t-1}$ and $u^{t-1}$, the corresponding correlation weight $c[t-1]$, and the connection status for the subvector $v^{t-2}$. Similarly, other subvectors of $u$ and $w$ and the corresponding correlation weights can be obtained.

3.3.3. Recombination. All subvectors of $u$ and $w$ can be obtained by lookup tables, and the $n$-bit $u$ and $w$ can be obtained by bit concatenation. The correlation weight of the modular addition is the sum of the weight of each subblock, i.e., $C w=\sum_{k=0}^{t-1} c[k]$.

When there are multiple modular additions in the round function, i.e., $N_{A}>1$, for each modular addition, its undetermined input mask and output mask need to be obtained by the Splitting-Lookup-Recombination approach, respectively. In the lookup phase, a total of $t N_{A}$ lookup 
(1) for each $b \in\{0,1\}$ and input mask $v \in \mathbb{F}_{2}^{m}$

(2) $\quad \operatorname{cLAT}_{\min }[v][b]=m$, let $M^{T}[k]=0$ and $\operatorname{cLAT}_{N}[v][b][k]=0$, for $0 \leq k \leq m-1$;

(3) for each input mask $w \in \mathbb{F}_{2}^{m}$ and output mask $u \in \mathbb{F}_{2}^{m}$ do

(4) $\quad A=u \oplus v, B=u \oplus w, C=u \oplus v \oplus w, C w=0$;

(5) for $j=0$ to $m-1$ do

(6) $\quad C_{b}[j]=(C \gg(m-1-j)) \wedge 1$;

(7) end for

(8) if $b=1$ then //determining the connection status generated by the upper subblock.

(30) end for.

else $C w++, M^{T}[0]=1, Z=1 \ll(m-1) ;$

end if $M^{T}[0]=0, Z=0 ;$

for $i=1$ to $m-1$ do //determining the correlation weight.

$M^{T}[i]=\left(C_{b}[i-1]+M^{T}[i-1]\right) \wedge 1$

if $M^{T}[i]=1$ then end if

$C w++, Z=Z \vee(1 \ll(m-1-i))$;

end for

$F_{1}=A \wedge(\neg(A \wedge Z)), F_{2}=B \wedge(\neg(B \wedge Z))$;

if $F_{1}=0$ and $F_{2}=0$ then //judgment conditions $u \oplus v \preceq z$ and $u \oplus w \preceq z$.

$\operatorname{cLAT}_{w}[v][b]\left[\operatorname{cLAT}_{N}[v][b][C w]\right]=w$;

$\operatorname{cLAT}_{u}[v][b]\left[\operatorname{cLAT}_{N}[v][b][C w]\right]=u$;

$\operatorname{cLAT}_{N}[v][b][C w]++; / /$ the number of tuples correspond to $v$ and $b$.

$\operatorname{cLAT}_{b}[u][v][w][b]=\left(M_{T}[m-1]+C_{b}[m-1]\right) \wedge 1 ; / /$ connection status.

if $\operatorname{cLAT}_{\min }[v][b]>C w$ then

$\operatorname{cLAT}_{\text {min }}[v][b]=C w$; //the minimum correlation weight corresponds to $v$ and $b$. end if

end if

end for

Algorithm 2: Constructing the $m$-bit cLAT for modular addition.

operations are required. And the correlation weight of the round function is $C w=\sum_{j=1}^{N_{A}} \sum_{k=0}^{t-1} c_{j}[k]$.

For each subvector $v^{k}$, the possible minimum linear correlation weight corresponding to it can be calculated in advance by Algorithm 2, that is,

$$
c[k]_{\min }=\min \left(\operatorname{cLAT}_{\min }\left[v^{k}\right][0], \operatorname{cLAT}_{\min }\left[v^{k}\right][1]\right) \text {. }
$$

During the Recombination phase, the correlation boundary can be constructed by the associated weights that have been obtained and the possible minimum correlation weights, shown in Corollary 2.

Corollary 2. For addition modulo $2^{n}$, one of the input mask $v=v^{t-1}\|\cdots\| v^{0}$ is fixed, $n=m t, v^{k} \in \mathbb{F}_{2}^{m}$, and $0 \leq k \leq t-1$. For any $u, w \in \mathbb{F}_{2}^{n}$ of nonzero correlation, the correlation boundary should have

$$
\begin{aligned}
\operatorname{Cor}(u, v, w) \leq & \operatorname{Cor}\left(u^{t-1}\|\cdots\| u^{k}, v^{t-1}\|\cdots\| v^{k}, w^{t-1}\|\cdots\| w^{k}\right) \\
& +2^{-\sum_{j=0}^{k-1} c[j]_{\min }} .
\end{aligned}
$$

Proof. The correlation of modular addition is the product of the correlation of each subblock after splitting, i.e., $\operatorname{Cor}(u, v, w)=\prod_{k=0}^{t-1} \operatorname{Cor}\left(u^{k}, v^{k}, w^{k}\right)$. Let $\quad-\log _{2} \operatorname{Cor}\left(u^{t-1}\right.$ $\left.\|\cdots\| u^{k}, v^{t-1}\|\cdots\| v^{k}, w^{t-1}\|\cdots\| w^{k}\right)=\sum_{l=k}^{t-1} c[l]$ be the correlation weight of the subvector tuples that are obtained by lookup tables. The sum of the correlation weights of the subvector tuples have not been looked up yet, which should s.t. $\sum_{j=0}^{k-1} c[j]_{\min } \leq-\log _{2} \operatorname{Cor}(u, v, w)-\sum_{l=k}^{t-1} c[l]$.

Assuming the number of $\left(u^{k}, w^{k}\right)$ corresponding to each subvector $v^{k}$ is $X_{k}$, hence, the number of mask branches corresponding to the modular addition is $\prod_{k=0}^{t-1} X_{k}$. Corollary 2 can be used to filter out $(u, w)$ of large correlation weight.

3.4. Improved Automatic Search Algorithm. In this section, we adopt a top-down technique [19-21], taking the first round as the starting point of the search process. In the first/second rounds, the input-output mask tuples of each modular addition with correlation weight increasing monotonically can be obtained by Algorithm 1 . In the middle rounds, for each modular addition, $u$ and $w$ can be obtained by the Splitting-Lookup-Recombination approach. Algorithm 3 takes SPECK as an example.

Let the optimal correlation weight of the $(r-i)^{\text {th }}$ round that has been obtained be $B c_{r-i}, 1 \leq i \leq r-1$, and let the expected $r$-round correlation weight be $\overline{B c_{r}}$. The correlation weight of the first two rounds should subject to Matsui's 
pruning condition, i.e., $C w_{1}+B c_{r-1} \leq \overline{B c_{r}}$ and $C w_{1}+C w_{2}+$ $B c_{r-2} \leq \overline{B c_{r}}$.

Let $\sum_{i=1}^{N_{A}^{\prime}} C w_{i}=\overline{B c_{r}}-B c_{r-1}, N_{A}^{\prime}$ is the number of additions in the first two rounds, and then the search space need to be constructed is no more than (19). When the block size of a ARX cipher is large, let $n$ be the word size of the modular addition, the total input-output masks of all $N_{A}^{\prime}$ modular additions in the first two round is $S^{\prime}=2^{3 n \times N_{A}^{\prime}}$. Therefore, when the value of $\overline{B c_{r}}-B c_{r-1}$ is small, the search space $S$ will be much smaller than $S^{\prime}$ intuitively:

$$
S=\prod_{i=1}^{N_{A}^{\prime}} \sum_{c=0}^{C w_{i}} \#\left\{(u, v, w) \mid-\log _{2} \operatorname{Cor}(u, v, w)=c\right\} .
$$

3.5. Flexible Search Scenario Settings. Combining Algorithm 1, cLAT, pruning conditions, and the properties of the target ciphers in Section 2.3, Algorithm 3 can be adapted to the following search scenarios with appropriate modifications:

Scenario 1: for some ARX ciphers, such as SPARX and Chaskey, the number of modular additions in the round function is more than $1, N_{A}>1$. Hence, the correlation weight of each round is $C w_{i}=\sum_{j=1}^{N_{A}} C w_{i}^{j}$, and $C w_{i}^{j}$ is the correlation weight of the $j^{\text {th }}$ addition in the $i^{\text {th }}$ round. In the first/second round, the inputoutput masks of each modular addition should be generated by Algorithm 1. In the middle rounds, the tuple $(u, w)$ of each modular addition should be obtained by calling $\operatorname{LR}(v)$ multiple times.

Scenario 2: the linear hulls can also be searched by simply modifying Algorithm 3. For an obtained optimal linear trail, fix the input mask of the first round and the output mask of the last round, call round- $r(i, x, y)$ directly, and modify $\overline{B c_{r}}$ to the expected maximum statistical correlation weight $C_{\max }$. Therefore, for ALP, all linear trails with linear correlation weight between the optimal correlation weight $C_{\min }$ and $C_{\max }$ can be counted.

Scenario 3: when the number of rounds of a linear trail or the block size is large, the linear correlation tends to be very small, and the search process will be very timeconsuming. Hence, good linear characteristic results under certain conditions can be explored by the heuristic search settings. We can exclude a large number of search branches and reduce the search complexity by these methods, such as starting the search from a desired large correlation weight $\overline{B c_{r}}$, fixing the input mask of a certain round, and limiting the correlation weight of a round or a certain modular addition.

\section{Applied to SPECK, SPARX, Chaskey, and CHAM-64}

4.1. Linear Hulls for SPECK32/48/64. Applying Algorithm 3, the optimal linear trails for SPECK32/48/64 with correlation close to $2^{-n / 2}$ can be obtained, shown in Table 2. Fixing the input and output masks, the ALP of the linear hulls obtained by the cluster experiment are given in Table 3. For SPECK64, new 14-round linear hull with an average linear potential of $2^{-61.24}$ has been found.

When searching for the linear hulls, we need to modify Algorithm 3 to adapt to Scenario 2. We use formula (13) to count ALP, where $C_{\min } \leq C w \leq C_{\max }, C_{\min }$ is the correlation weight of the linear trail we choose to use to pin the input and output masks, and $C_{\max }$ is the maximum correlation weight we limit our search. In the middle rounds, we adopt $C_{\max }$ to instead $\overline{B c_{r}}$ for filtering out those trails that contribute less to the ALP.

4.2. Linear Characteristics for SPARX. Searching for the optimal linear trails of SPARX variants, Algorithm 3 needs to be modified to fit Scenario 1. There are multiple modular additions in each round, $N_{A}=2$ for SPARX-64 and $N_{A}=4$ for SPARX-128. Hence, for all $2 N_{A}$ additions modulo $2^{16}$ in the first/second rounds, call Algorithm 1 for each addition to generate its input-output mask tuples, and then apply Property 3/4 to obtain the masks of the first two rounds for SPARX-64 and SPARX-128, respectively. In the middle rounds, the Splitting-Lookup-Recombination method is applied to obtain possible input masks and output masks of each addition, as well as correlation weight. The 10-round optimal linear trails for SPARX-64 are listed in Table 4, and the 11-round linear trail is obtained by limiting the correlation weight of each modular addition less than 3 in the first two rounds. Fixing the input and output mask of the 10round optimal linear trail, a 10-round linear hull with an ALP of $2^{-40.92}$ is obtained.

For SPARX-128, the first 8-round optimal linear trails can be derived from the first 8-round optimal linear trails of SPECK32 when considering the minimum active ARX-box. The experiment results in Table 5 confirmed the derivation. Based on Scenario 3, we limit the correlation weight of each modular addition in the first/second round to less than 2 , then we get the 9/10round linear trails with a correlation weight of $18 / 23$. Although the 9/10-round linear trail cannot be guaranteed as the best, they can still be used to get the 9/10round linear hulls with the corresponding ALP of $2^{-35.22} / 2^{-46}$.

The 11/10-round linear trails for SPARX-64/SPARX128 are given by Table $6 . c_{j}^{r}$ represents the correlation weight of the $j^{\text {th }}$ modular addition in the $r^{\text {th }}$ round, and $C w_{r}$ represents the correlation weight of the $r^{\text {th }}$ round, $0 \leq j<N_{A}$.

4.3. Linear Characteristics for Chaskey. From Table 7, the correlation weights of the first 3-round optimal linear trails of Chaskey we have found are $0 / 2 / 9$. For one-round optimal linear trail with a correlation weight of 0 , inputoutput masks represented in hexadecimal are $(1,1,0,0)$ and $(0,80,800000,0)$. To find the linear trails with longer rounds, we use a heuristic approach in Scenario 3, limiting the correlation weight of each modular additions in the first round to less than 2 and setting the correlation weight 
Input: the cLAT is precomputed and stored by Algorithm 2. $B w_{1}, \cdots, B w_{r-1}$ have been recorded

(1) Program entry:

(2) Let $\overline{B c_{r}}=B c_{r-1}-1$, and $B c_{r}=$ null $/ / B w_{1}$ can be derived manually for most ARX ciphers.

(3) while $\overline{B c_{r}} \neq B c_{r}$ do

(4) $\overline{B c_{r}}++$; //the expected $r$-round correlation weight increases monotonously.

(5) Call Procedure Round-1;

(6) end while

(7) Exit the program.

(8) Round-1:/exclude the search space with correlation weights larger than $\overline{B c_{r}}-B c_{r-1}$.

(9) for $C w_{1}=0$ to $n-1$ do $/ / C w_{1}$ increases monotonously.

(10) if $C w_{1}+B c_{r-1}>\overline{B c_{r}}$ then

(11) Return to the upper procedure with FALSE state;

(12) else

(13) Call Algorithm 1 Const $\left(S_{C w_{1}}\right)$, and traverse each output tuple $\left(u_{1}, v_{1}, w_{1}\right)$;

(14) if call Round-2 $\left(u_{1}, v_{1}, w_{1}\right)$ and the return value is TRUE, then

(18) end for

(19) Return to the upper procedure with FALSE state;

(20) Round-2 $\left(u_{1}, v_{1}, w_{1}\right)$ : /exclude the correlation weights larger than $\overline{B c_{r}}-B c_{r-1}-C w_{1}$.

(21) for $C w_{2}=0$ to $n-1 / / C w_{2}$ increases monotonously.

(32) Return to the upper procedure with FALSE state;

(33) Round- $r(i, x, y)$ : //middle rounds, $3 \leq i \leq r$.

(34) $v=x \ggg r_{a}$, and let $v=v^{t-1}\|\cdots\| v^{0}$ and $v^{k} \in \mathbb{F}_{2}^{m}, 0 \leq k \leq t-1$; //splitting $v$.

(35) Call $L R(v)$, traversing each $u$ and $w$; //where $C w_{i}=\operatorname{Cor}(v, w, u)$.

(36) if $i=r$ and $C w_{1}+\ldots+C w_{i-1}+C w_{i}=\overline{B c_{r}}$ then //the last round.

(37) Let $B c_{r}=\overline{B c_{r}}$, break from $\operatorname{LR}(v)$ and return TRUE;

(38) end if $/ / r$-round optimal linear trail of expected correlation weight $\overline{B c_{r}}$ have been found.

(39) $y^{\prime}=(y \oplus w) \lll r_{b}, x^{\prime}=y^{\prime} \oplus u$;

(40) if call Round $-r\left(i+1, x^{\prime}, y^{\prime}\right)$ and the return value is TRUE, then

(41) Break from $L R(v)$ and return TRUE; //record the masks of each round and return.

(42) end if

(43) Return to the upper procedure with FALSE state;

(44) LR $(v)$ : //looking up cLAT and recombining another input mask $w$ and the output mask $u$.

(45) Let $c[k]_{\min }=\min \left(\operatorname{cLAT}_{\min }\left[v^{k}\right][0], \operatorname{cLAT}_{\min }\left[v^{k}\right][1]\right)$, and $b[k]=0$, for $0 \leq k \leq t-1$;

(46) for $k=t-1$ to 0 do //from MSB to LSB direction.

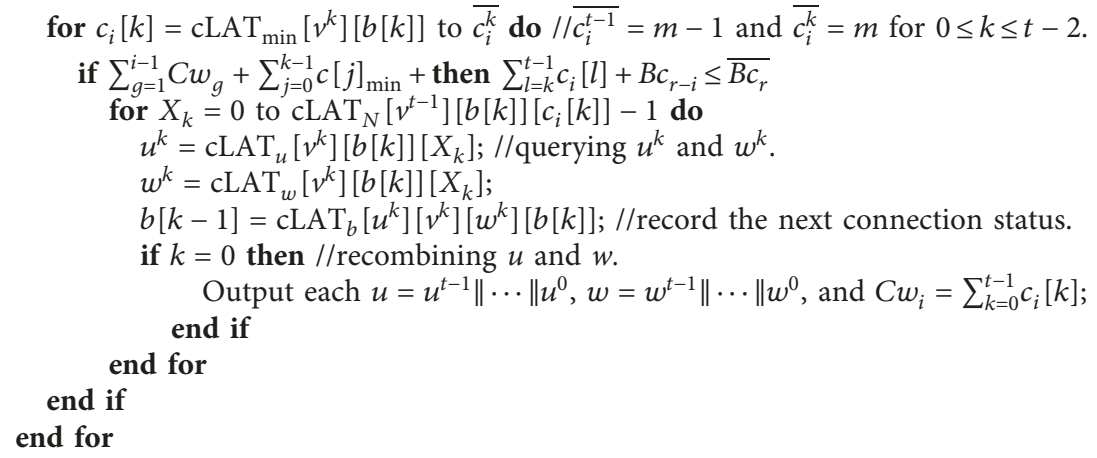


TABLE 2: The 9/10/13 round optimal linear trails for SPECK32/48/64.

\begin{tabular}{|c|c|c|c|c|c|c|}
\hline \multirow{2}{*}{$r$} & \multicolumn{2}{|c|}{ SPECK32 } & \multicolumn{2}{|c|}{ SPECK48 } & \multicolumn{2}{|c|}{ SPECK64 } \\
\hline & $\Gamma X_{r}$ & $C w_{r}$ & $\Gamma X_{r}$ & $C w_{r}$ & $\Gamma X_{r}$ & $C w_{r}$ \\
\hline 0 & $00 \mathrm{~A} 0062 \mathrm{~F}$ & 1 & 000180B80001 & 1 & 0100012014010021 & 2 \\
\hline 1 & 78B818B9 & 4 & $000000 \mathrm{C} 00001$ & 0 & 0001810020000101 & 1 \\
\hline 2 & 00906021 & 1 & 00000E00000 E & 2 & 0000010000000001 & 0 \\
\hline 3 & 60804081 & 1 & 7A0070700070 & 5 & 0000000100000000 & 1 \\
\hline 4 & 00800001 & 0 & C2C080829380 & 6 & 0D0000000C000000 & 2 \\
\hline 5 & 00010000 & 1 & D00000108300 & 2 & $60610000606 \mathrm{C} 0000$ & 3 \\
\hline 6 & 0B000800 & 3 & 800000809800 & 1 & 00024D0300620C03 & 6 \\
\hline 7 & 20402050 & 2 & $00000400 \mathrm{C} 004$ & 1 & $181070141 \mathrm{~B} 107358$ & 6 \\
\hline 8 & 008380C3 & 1 & 200020260020 & 2 & 0013001818031840 & 3 \\
\hline 9 & $170 \mathrm{~B} 130 \mathrm{~A}$ & - & 013100310100 & 2 & 1818000000181200 & 2 \\
\hline 10 & & & 8800A8880109 & - & 0018000000001000 & 1 \\
\hline 11 & & & & & 0000100000000000 & 1 \\
\hline 12 & & & & & 0000009800000080 & 2 \\
\hline 13 & & & & & 5000040480000404 & - \\
\hline
\end{tabular}

TABLE 3: Linear hulls for SPECK32/48/64.

\begin{tabular}{|c|c|c|c|c|c|c|c|c|c|}
\hline $2 n$ & $r$ & $\Gamma_{\text {in }}$ & $\Gamma_{\text {out }}$ & $C_{\min }$ & $C_{\max }$ & ALP & No. of trails & Time & Reference \\
\hline \multirow{3}{*}{32} & 9 & 0010,1400 & 0B00, 0800 & 15 & 25 & $2^{-29.1}$ & 69737 & N/A & [18] \\
\hline & 9 & 0380,5224 & $066 \mathrm{~A}, 0608$ & 14 & 14 & $2^{-28}$ & 1 & $\mathrm{~N} / \mathrm{A}$ & [16] \\
\hline & 9 & $00 \mathrm{~A} 0,062 \mathrm{~F}$ & $170 \mathrm{~B}, 130 \mathrm{~A}$ & 14 & 20 & $2^{-27.78}$ & 14 & $25 \mathrm{~s}$ & This paper \\
\hline \multirow{3}{*}{48} & 10 & 000131,050021 & 2484F2, 2480F6 & 22 & N/A & $2^{-44}$ & 1 & N/A & {$[16]$} \\
\hline & 10 & 800121,158021 & DE84DC, C684DC & 22 & N/A & $2^{-44}$ & 1 & $\mathrm{~N} / \mathrm{A}$ & [18] \\
\hline & 10 & $000180, \mathrm{~B} 80001$ & 8800A8, 880109 & 22 & 28 & $2^{-43.64}$ & 50 & $157.3 \mathrm{~h}$ & This paper \\
\hline \multirow{4}{*}{64} & 13 & 18600010,10724800 & 00024982,00420802 & 30 & 30 & $2^{-60}$ & 1 & $\mathrm{~N} / \mathrm{A}$ & {$[16]$} \\
\hline & 13 & 00101800,00001812 & 00006065,00006068 & 30 & 30 & $2^{-60}$ & 1 & $\mathrm{~N} / \mathrm{A}$ & [18] \\
\hline & 13 & 01000120,14010021 & 50000404,80000404 & 30 & 32 & $2^{-55.29}$ & 178 & $7.3 \mathrm{~h}$ & This paper \\
\hline & 14 & 01000120,14010021 & 26902000,20802006 & 33 & 35 & $2^{-61.24}$ & 194 & $5.8 \mathrm{~h}$ & This paper \\
\hline
\end{tabular}

All experiment code in this work are run on a single high-performance server with Intel(R) Xeon(R) CPU E5-2680 v4 @ 2.40 GHz. All masks are represented in hexadecimal.

TABLE 4: Linear characteristics for SPARX-64.

\begin{tabular}{ccccccccccccccccc}
\hline$r$ & $C w$ & $T_{\text {trail }}$ & \multicolumn{4}{c}{$\Gamma_{\text {in }}$} & \multicolumn{4}{c}{$\Gamma_{\text {out }}$} & & $C_{\min }$ & $C_{\max }$ & ALP & No. of trails & $T_{\text {ALP }}$ \\
\hline 1 & 0 & $0 \mathrm{~s}$ & 0000 & 0000 & 0080 & 4001 & 0000 & 0000 & 0000 & 0001 & 0 & 0 & 1 & 1 \\
2 & 0 & $0 \mathrm{~s}$ & 0000 & 0000 & 0080 & 4001 & 0000 & 0000 & 0004 & 0004 & 0 & 0 & 1 & 1 \\
3 & 1 & $0 \mathrm{~s}$ & 0000 & 0000 & 0080 & 4001 & $2 \mathrm{C} 10$ & 2010 & $2 \mathrm{C} 1 \mathrm{C}$ & $201 \mathrm{C}$ & 1 & 10 & $2^{-2}$ & 1 \\
4 & 3 & $0 \mathrm{~s}$ & 0090 & 6021 & 0000 & 0000 & 0000 & 0000 & $0 \mathrm{~B} 00$ & 0800 & 3 & 10 & $2^{-6}$ & 1 & $0 \mathrm{~s}$ \\
5 & 5 & $0 \mathrm{~s}$ & $00 \mathrm{~A} 0$ & 3021 & 0000 & 0000 & 0000 & 0000 & $85 \mathrm{C} 2$ & 8442 & 5 & 10 & $2^{-9.98}$ & 3 & $3 \mathrm{~s}$ \\
6 & 7 & $0 \mathrm{~s}$ & 0080 & 5021 & 0000 & 0000 & 4285 & 4284 & 4385 & 4384 & 7 & 15 & $2^{-13.91}$ & 11 & $22 \mathrm{~s}$ \\
7 & 11 & $3.2 \mathrm{~m}$ & 0090 & 6021 & 0000 & 0000 & $170 \mathrm{~B}$ & $130 \mathrm{~A}$ & $17 \mathrm{CF}$ & $130 \mathrm{~F}$ & 11 & 17 & $2^{-21.87}$ & 5 & $9 \mathrm{~s}$ \\
8 & 15 & $4.6 \mathrm{~h}$ & 0880 & $503 \mathrm{D}$ & 0090 & 6021 & 0000 & 0000 & $0 \mathrm{E} 81$ & $1 \mathrm{~EB} 0$ & 15 & 20 & $2^{-29.73}$ & 22 & $2 \mathrm{~m}$ \\
9 & 19 & $48.6 \mathrm{~h}$ & 0080 & 5021 & 2000 & 0058 & 2058 & 2040 & 3858 & 3840 & 19 & 25 & $2^{-35.97}$ & 1215 & $28.7 \mathrm{~h}$ \\
10 & 22 & $3 \mathrm{~d}$ & 0080 & 4001 & $00 \mathrm{C} 0$ & $\mathrm{~F} 001$ & 0205 & 0204 & 0205 & 0204 & 22 & 25 & $2^{-40.92}$ & 144 & $1 \mathrm{~h}$ \\
11 & $\leq 28$ & $5 \mathrm{~m}$ & 0080 & 4001 & $00 \mathrm{C} 0$ & F001 & $38 \mathrm{~A} 8$ & 2080 & 2058 & 2040 & 28 & 28 & $2^{-56}$ & 1 & - \\
\hline
\end{tabular}

to the expected values to start the heuristic search. The 4/ 5-round linear trails with correlation weights of 29/61 are obtained. The details of the linear trails are listed in Table 8.

4.4. Linear Characteristics for CHAM-64. In the first 4 rounds of CHAM, the input-output mask tuples of each modular addition can be constructed by Algorithm 1, and all input and output masks for the first 4 rounds are deduced by Property 5. In the forward search process, the Splitting-Lookup-Recombination approach is adopted to determine the possible unknown input masks and output masks for the modular addition in each round. In [7], for CHAM-64, a 34-round linear trail with a bias of $\varepsilon=2^{-31}$ was given. The correlation weights of the optimal linear trails obtained by us are shown in Table 9. The correlation of the 34 -round optimal linear trail we find is $2^{-31}$, and the 
TABLE 5: Linear characteristics for SPARX-128.

\begin{tabular}{|c|c|c|c|c|c|c|c|c|c|}
\hline$r$ & $C w$ & $T_{\text {trail }}$ & $\Gamma_{\text {in }}$ & $\Gamma_{\text {out }}$ & $C_{\min }$ & $C_{\max }$ & ALP & No. of trails & $T_{\text {hull }}$ \\
\hline \multirow[t]{2}{*}{1} & 0 & $0 \mathrm{~s}$ & 0000000000000000 & 0000000000000000 & & & & & \\
\hline & & & 0000000000804001 & 0000000000000001 & 0 & 0 & 1 & 1 & $0 \mathrm{~s}$ \\
\hline \multirow[t]{2}{*}{2} & 0 & $0 \mathrm{~s}$ & 0000000000000000 & 0000000000000000 & & & & & \\
\hline & & & 0000000000804001 & 0000000000040004 & 0 & 0 & 1 & 1 & $0 \mathrm{~s}$ \\
\hline \multirow[t]{2}{*}{3} & 1 & $0 \mathrm{~s}$ & 0000000000000000 & 0000000000000000 & & & & & \\
\hline & & & 0000000000804001 & $000000002 \mathrm{C} 102010$ & 1 & 3 & $2^{-2}$ & 1 & $0 \mathrm{~s}$ \\
\hline \multirow[t]{2}{*}{4} & 3 & $0 \mathrm{~s}$ & 0000000000000000 & $000000000 \mathrm{~B} 000800$ & & & & & \\
\hline & & & 0000000000906021 & 0B03000300030803 & 3 & 10 & $2^{-6}$ & 1 & $0 \mathrm{~s}$ \\
\hline \multirow[t]{2}{*}{5} & 5 & $2 \mathrm{~s}$ & 0000000000A03021 & 0000000000000000 & & & & & \\
\hline & & & 0000000000000000 & $0000000085 \mathrm{C} 28442$ & 5 & 10 & $2^{-9.98}$ & 3 & $0 \mathrm{~s}$ \\
\hline \multirow[t]{2}{*}{6} & 7 & $1 \mathrm{~s}$ & 0000000000805021 & 0000000000000000 & & & & & \\
\hline & & & 0000000000000000 & 0000000042854284 & 7 & 12 & $2^{-13.91}$ & 3 & $1 \mathrm{~s}$ \\
\hline \multirow[t]{2}{*}{7} & 9 & $4 \mathrm{~s}$ & 0000000000906021 & 0000000000000000 & & & & & \\
\hline & & & 0000000000000000 & $00000000170 \mathrm{~B} 130 \mathrm{~A}$ & 9 & 15 & $2^{-17.78}$ & 5 & $13 \mathrm{~s}$ \\
\hline \multirow[t]{2}{*}{8} & 12 & $13 \mathrm{~d}$ & 0000000000811381 & 00000000170B130A & & & & & \\
\hline & & & 0000000000000000 & $160 \mathrm{~F} 01040104120 \mathrm{E}$ & 12 & 18 & $2^{-23.65}$ & 11 & $101 \mathrm{~s}$ \\
\hline \multirow[t]{2}{*}{9} & $\geq 14$ & - & 0000000000004020 & 0000000002150012 & & & & & \\
\hline & $\leq 18$ & $27 \mathrm{~m}$ & 0000000000000000 & 081308140A060806 & 18 & 22 & $2^{-35.22}$ & 53 & $6 \mathrm{~h}$ \\
\hline \multirow[t]{2}{*}{10} & $\geq 18$ & - & 0000000000804001 & 000000000B810800 & & & & & \\
\hline & $\leq 23$ & $4.4 \mathrm{~d}$ & 0000000000000000 & 09840A0402050204 & 23 & 23 & $2^{-46}$ & 1 & - \\
\hline
\end{tabular}

TABLE 6: The 11/10-round linear trails for SPARX-64/SPARX-128.

\begin{tabular}{|c|c|c|c|c|c|c|c|c|c|c|c|}
\hline \multirow[b]{2}{*}{$r$} & \multicolumn{4}{|c|}{ 11-round trail for SPARX-64 } & \multicolumn{7}{|c|}{ 10-round trail for SPARX-128 } \\
\hline & $\Gamma X_{0}^{r}\|\cdots\| \Gamma X_{0}^{r}$ & $c_{0}^{r}$ & $c_{1}^{r}$ & $C w_{r}$ & $r$ & $\Gamma X_{0}^{r}\left\|\Gamma X_{1}^{r}\right\| \cdots\left\|\Gamma X_{6}^{r}\right\| \Gamma X_{7}^{r}$ & $c_{0}^{r}$ & $c_{1}^{r}$ & $c_{2}^{r}$ & $c_{3}^{r}$ & $C w_{r}$ \\
\hline 1 & 0080400100C0F001 & 0 & 1 & 1 & 1 & 00000000000402000000000000000000 & 0 & 0 & 0 & 0 & 0 \\
\hline 2 & $000000010000 \mathrm{C} 001$ & 0 & 0 & 0 & 2 & 00000000081008100000000000000000 & 0 & 1 & 0 & 0 & 1 \\
\hline 3 & 0004000400070007 & 1 & 2 & 3 & 3 & 000000000C010E000000000000000000 & 0 & 3 & 0 & 0 & 3 \\
\hline $\mathcal{L}$ & 2C1020102C1C201 C & - & - & - & 4 & 00000000364834500000000000000000 & 0 & 5 & 0 & 0 & 5 \\
\hline 4 & 2C1C201C00000000 & 4 & 0 & 4 & $\mathcal{L}^{\prime}$ & 00000000008 A $90 \mathrm{E} 20000000000000000$ & - & - & - & - & - \\
\hline 5 & 3140013000000000 & 3 & 0 & 3 & 5 & 000000000000000000000000008A90E2 & 0 & 0 & 0 & 2 & 2 \\
\hline 6 & 858D05CF00000000 & 4 & 0 & 4 & 6 & $000000000000000000000000078 \mathrm{~F} 138 E$ & 0 & 0 & 0 & 4 & 4 \\
\hline $\mathcal{L}$ & 60117B1800000000 & 一 & - & - & 7 & $000000000000000000000000000 \mathrm{E} 1600$ & 0 & 0 & 0 & 2 & 2 \\
\hline 7 & $0000000060117 \mathrm{~B} 18$ & 0 & 4 & 4 & 8 & 00000000000000000000000000001800 & 0 & 0 & 0 & 0 & 0 \\
\hline 8 & 0000000000812261 & 0 & 3 & 3 & $\mathcal{L}^{\prime}$ & 00000000000000000000000060006000 & - & - & - & - & - \\
\hline 9 & 0000000080018280 & 0 & 1 & 1 & 9 & 00000000600060006000000000006000 & 0 & 1 & 1 & 0 & 2 \\
\hline $\mathcal{L}$ & 0000000000020202 & - & - & - & 10 & 00000000828182010280020080018001 & 0 & 2 & 1 & 1 & 4 \\
\hline 10 & 0002020200000200 & 1 & 0 & 1 & 11 & 0000000002150012081308140 A060806 & - & - & - & - & - \\
\hline 11 & $1 \mathrm{E} 08180808000800$ & 3 & 1 & 4 & & & & & & & \\
\hline 12 & 38A8208020582040 & - & - & - & & & & & & & \\
\hline
\end{tabular}

TABle 7: Correlation of the linear trails for Chaskey.

\begin{tabular}{lcccccc}
\hline Round & 1 & 2 & 3 & 4 & 5 & Reference \\
\hline Correlation & $2^{-1}$ & $2^{-2}$ & $2^{-9}$ & - & - & {$[18]$} \\
Correlation & 1 & $2^{-2}$ & $2^{-9}$ & $\geq 2^{-29}$ & $\geq 2^{-61}$ & This paper \\
\hline
\end{tabular}

details of the 35-round optimal linear trail with a correlation of $2^{-33}$ are listed in Table 10 .

\section{Conclusions}

In this paper, we have improved the automatic search algorithm for the linear characteristics on ARX ciphers. Combining with the optimization strategies of constructing the input-output masks corresponding to specific correlation weight and the novel concept of cLAT, this search tool enables an efficient search for the linear characteristics on typical ARX ciphers. Applying this tool, we get new 9/ 10/14-round linear hulls for SPECK32/48/64, and the ALP is $2^{-27.78}, 2^{-43.64}$, and $2^{-61.24}$, respectively. For SPARX-64, a 10-round optimal linear trail with a correlation of $2^{-22}$ and a 11-round good linear trail with a correlation of $2^{-28}$ have been obtained. For SPARX-128, a 10-round linear trail with a correlation of $2^{-23}$ is obtained. The linear cryptanalysis results on SPARX are presented for the first time so far. For Chaskey, the linear characteristic results have been 
TABLE 8: Linear trails for Chaskey.

\begin{tabular}{|c|c|c|c|c|c|c|c|c|c|c|}
\hline \multirow{2}{*}{$r$} & \multicolumn{5}{|l|}{2 rounds with Cor $=2^{-2}$} & \multicolumn{5}{|l|}{3 rounds with Cor $=2^{-9}$} \\
\hline & $a, b, c, d$ & $c_{0}^{r}$ & $c_{1}^{r}$ & $c_{2}^{r}$ & $c_{3}^{r}$ & $a, b, c, d$ & $c_{0}^{r}$ & $c_{1}^{r}$ & $c_{2}^{r}$ & $c_{3}^{r}$ \\
\hline 0 & 2000001, B100001, 1, 1 & 1 & 0 & 0 & 0 & $1,8100001,201,303$ & 0 & 1 & 0 & 1 \\
\hline 1 & $0,1,0,0$ & 0 & 0 & 0 & 1 & $300,1,0,0$ & 5 & 0 & 0 & 0 \\
\hline 2 & $300004,1000,10000000,4$ & - & - & - & - & $0,10000,1,0$ & 0 & 0 & 1 & 1 \\
\hline 3 & & & & & & $240030,10008080,81011000,240000$ & - & - & - & - \\
\hline & 4 rounds with Cor $=2^{-29}$ & & & & & 5 rounds with Cor $=2^{-61}$ & & & & \\
\hline$r$ & $a, b, c, d$ & $c_{0}^{r}$ & $c_{1}^{r}$ & $c_{2}^{r}$ & $c_{3}^{r}$ & $a, b, c, d$ & $c_{0}^{r}$ & $c_{1}^{r}$ & $c_{2}^{r}$ & $c_{3}^{r}$ \\
\hline 0 & $0,800,90001,10 \mathrm{D} 0801$ & 0 & 2 & 1 & 0 & $1,8100001,201,303$ & 0 & 1 & 0 & 1 \\
\hline 1 & $0,0,1,1$ & 0 & 0 & 0 & 0 & $300,1,0,0$ & 5 & 0 & 0 & 0 \\
\hline 2 & $0,80,810000,0$ & 0 & 2 & 3 & 1 & $0,10000,1,0$ & 0 & 0 & 1 & 1 \\
\hline 3 & $18001 \mathrm{~A} 20,80880040,400189,1 \mathrm{~A} 20$ & 5 & 3 & 7 & 5 & $240030,10008080,81011000,240000$ & 4 & 4 & 6 & 6 \\
\hline 4 & 2D224005, A83F0DA8, 2DA1C86D, 25004107 & - & - & - & - & 50E73286, 8241A0, 5161469B, 40D436A6 & 10 & 12 & 4 & 6 \\
\hline 5 & & & & & & BAAE7E16, 76224512, 65104022, 3EA61E37 & - & - & 一 & - \\
\hline
\end{tabular}

TABLE 9: Correlation weights of the optimal linear trails for CHAM-64.

\begin{tabular}{lllllllllllllllllllllllllllllllllllll}
\hline Round & 1 & 2 & 3 & 4 & 5 & 6 & 7 & 8 & 9 & 10 & 11 & 12 & 13 & 14 & 15 & 16 & 17 & 18 & 19 & 20 & 21 & 22 & 23 & 24 & 25 & 26 & 27 & 28 & 29 & 30 & 31 & 32 & 33 & 34 & 35
\end{tabular} $\begin{array}{llllllllllllllllllllllllllllllllllll}\mathrm{Cw} & 0 & 0 & 0 & 0 & 0 & 0 & 0 & 1 & 2 & 3 & 3 & 4 & 5 & 6 & 7 & 9 & 10 & 12 & 14 & 16 & 17 & 18 & 20 & 21 & 22 & 23 & 25 & 26 & 26 & 26 & 27 & 28 & 29 & 31 & 33\end{array}$

TABLE 10: 35-round optimal linear trail for CHAM-64.

\begin{tabular}{lcccc}
\hline$r$ & $\Gamma X_{0}^{r}\|\cdots\| \Gamma X_{3}^{r}$ & $C w_{r}$ & $r$ & $\Gamma X_{0}^{r}\|\cdots\| \Gamma X_{3}^{r}$ \\
\hline 1 & 0000000000018000 & 0 & 19 & $0007010380 C 08000$ \\
2 & 0000000180000000 & 0 & 20 & $810080 C 080000700$ \\
3 & 0001800000000000 & 0 & 21 & 0001800007000201 \\
4 & 0000000000000100 & 0 & 22 & 0000070002010100 \\
5 & 0000000001000000 & 0 & 23 & 0700020101000000 \\
6 & 0000010000000000 & 0 & 24 & 0001010000000006 \\
7 & 0100000000000000 & 1 & 25 & 0000000000060002 \\
8 & $00 C 0000000000001$ & 1 & 26 & 0000000600020000 \\
9 & 8000000000010100 & 1 & 27 & 0006000200000000 \\
10 & $40000001010000 \mathrm{C} 0$ & 1 & 28 & 0000000000000400 \\
11 & $0061010000 \mathrm{C} 08000$ & 1 & 29 & 0000000004000000 \\
12 & $812000 \mathrm{C} 080004100$ & 4 & 30 & 0000040000000000 \\
13 & 0041800041008201 & 1 & 31 & 04000000000000000 \\
14 & 0030410082014100 & 3 & 32 & 0200000000000006 \\
15 & 6500820141000060 & 3 & 33 & 0002000000060600 \\
16 & $\mathrm{~A} C 1410000600047$ & 3 & 34 & 0001000606000200 \\
18 & $\mathrm{C} 080006000470103$ & 2 & 35 & 0106060002000002 \\
\end{tabular}

updated, which cover more rounds than the existing results. For CHAM-64, the linear characteristics we obtained are the first third-party linear cryptanalysis results. In addition, we believe that these improved optimization strategies can also be achieved by linear cryptanalysis on other ARX ciphers.

\section{Data Availability}

The data obtained to support the findings of this work are available from the authors upon request. An extended version of this article can be found at https://eprint.iacr.org/2019/1319.

\section{Conflicts of Interest}

The authors declare that there are no conflicts of interest regarding the publication of this paper.

\section{Acknowledgments}

This work was supported by the National Key Research and Development Program of China (no. 2017YFB0801900).

\section{References}

[1] A. Biryukov and L. Perrin, "State of the art in lightweight symmetric cryptography," Cryptology ePrint Archive, vol. 2017, p. 511, 2017.

[2] D. Hong, J. Sung, S. Hong et al., "Hight: a new block cipher suitable for low-resource device," in Proceedings of the International Workshop on Cryptographic Hardware and Embedded Systems, pp. 46-59, Yokohama, Japan, October 2006.

[3] R. Beaulieu, D. Shors, J. Smith, S. Treatman-Clark, B. Weeks, and L. Wingers, The Simon and Speck Families of Lightweight 
Block Ciphers, Cryptology ePrint Archive, Report 2013/404, 2013, https://eprint.iacr.org/2013/404.

[4] D. Hong, J. Lee, D. Kim, D. Kwon, K. H. Ryu, and D. Lee, "LEA: a 128-bit block cipher for fast encryption on common processors," in Proceedings of the Information Security Applications-14th International Workshop, pp. 3-27, Jeju Island, Korea, 2013.

[5] N. Mouha, B. Mennink, A. V. Herrewege, D. Watanabe, B. Preneel, and I. Verbauwhede, "Chaskey: an efficient MAC algorithm for 32-bit microcontrollers," in Proceedings of the Selected Areas in Cryptography-SAC 2014-21st International Conference, pp. 306-323, Montreal, QC, Canada, August 2014.

[6] D. Dinu, L. Perrin, A. Udovenko, V. Velichkov, J. Großschädl, and A. Biryukov, "Design strategies for ARX with provable bounds: Sparx and LAX," in Proceedings of the International Conference on the Theory and Application of Cryptology and Information Security, pp. 484-513, Hanoi, Vietnam, December 2016.

[7] B. Koo, D. Roh, H. Kim et al., "A family of lightweight block ciphers for resource-constrained devices," in Proceedings of the Information Security and Cryptology-ICISC 2017-20th International Conference, pp. 3-25, Seoul, South Korea, December 2017.

[8] L. Song, Z. Huang, and Q. Yang, "Automatic differential analysis of ARX block ciphers with application to SPECK and LEA," in Proceedings of the Australasian Conference on Information Security and Privacy, pp. 379-394, Melbourne, VIC, Australia, July 2016.

[9] M. Matsui, "Linear cryptanalysis method for DES cipher," in Proceedings of the Advances in Cryptology-EUROCRYPT '93, Workshop on the Theory and Application of of Cryptographic Techniques, pp. 386-397, Lofthus, Norway, May 1993.

[10] M. Matsui, "On correlation between the order of s-boxes and the strength of DES," in Proceedings of the Advances in Cryptology-EUROCRYPT '94, Workshop on the Theory and Application of Cryptographic Techniques, pp. 366-375, Perugia, Italy, May 1994.

[11] H. Lipmaa, J. Wallén, and P. Dumas, "On the additive differential probability of exclusive-or," in Proceedings of the Fast Software Encryption, 11th International Workshop, pp. 317331, FSE 2004, Delhi, India, 2004.

[12] K. Nyberg and J. Wallén, "Improved linear distinguishers for SNOW 2.0," in Proceedings of the Fast Software Encryption, 13th International Workshop, FSE 2006, pp. 144-162, Gzustria, 2006.

[13] J. Wallén, "Linear approximations of addition modulo $2^{n}$," in Proceedings of the Fast Software Encryption, 10th International Workshop, FSE2003, pp. 261-273, Lund, Sweden, 2003.

[14] J. Wallén, "On the differential and linear properties of addition," Master's thesis, Helsinki University of Technology, Laboratory for Theoretical Computer Science, 2003, http:// www.tcs.hut.fi/Publications/bibdb/HUT-TCS-A84.pdf.

[15] Y. Yao, B. Zhang, and W. Wu, "Automatic search for linear trails of the SPECK family," in Proceedings of the Information Security-18th International Conference, ISC 2015, pp. 158-176, Trondheim, Norway, September 2015.

[16] K. Fu, M. Wang, Y. Guo, S. Sun, and L. Hu, "MILP-based automatic search algorithms for differential and linear trails for Speck," in Proceedings of the Fast Software Encryption-23rd International Conference, pp. 268-288, Bochum, Germany, 2016.

[17] E. Schulte-Geers, "On CCZ-equivalence of addition $\bmod 2^{n}$," Designs, Codes and Cryptography, vol. 66, no. 1-3, pp. 111127, 2013.
[18] Y. Liu, Q. Wang, and V. Rijmen, "Automatic search of linear trails in ARX with applications to Speck and Chaskey," in Proceedings of the Applied Cryptography and Network Security-14th International Conference, pp. 485-499, Guildford, UK, June 2016.

[19] A. Biryukov, V. Velichkov, and Y. Le Corre, "Automatic search for the best trails in ARX: application to block cipher Speck," in Proceedings of the International Conference on Fast Software Encryption, pp. 289-310, Bochum, Germany, March 2016.

[20] Z. Liu, Y. Li, and M. Wang, "Optimal differential trails in simon-like ciphers," Transactions on Symmetric Cryptology, vol. 2017, no. 1, pp. 358-379, 2017.

[21] Z. Liu, Y. Li, and M. Wang, "The security of simon-like ciphers against linear cryptanalysis," Cryptology ePrint Archive, vol. 2017, p. 576, 2017.

[22] S. Mella, J. Daemen, and G. V. Assche, "New techniques for trail bounds and application to differential trails in keccak," Transactions on Symmetric Cryptology, vol. 2017, no. 1, pp. 329-357, 2017.

[23] Y. Liu, K. Fu, W. Wang, L. Sun, and M. Wang, "Linear cryptanalysis of reduced-round SPECK," Information Processing Letters, vol. 116, no. 3, pp. 259-266, 2016.

[24] D. Bodden, "Linear cryptanalysis of reduced-round speck with a heuristic approach: automatic search for linear trails," in Proceedings of the Information Security-21st International Conference, ISC 2018, Guildford, UK, 2018.

[25] J. Daemen, R. Govaerts, and J. Vandewalle, "Correlation matrices," in Proceedings of the Fast Software Encryption: Second International Workshop, pp. 275-285, Leuven, Belgium, 1994.

[26] K. Nyberg, "Linear approximation of block ciphers," in Proceedings of the Advances in Cryptology-EUROCRYPT '94, Workshop on the Theory and Application of Cryptographic Techniques, pp. 439-444, Perugia, Italy, 1994.

[27] N. Mouha, "Chaskey: a MAC algorithm for microcontrollersstatus update and proposal of chaskey-12," IACR Cryptology ePrint Archive, vol. 2015, p. 1182, 2015.

[28] G. Ehrlich, "Loopless algorithms for generating permutations, combinations, and other combinatorial configurations," Journal of the ACM, vol. 20, no. 3, pp. 500-513, 1973. 


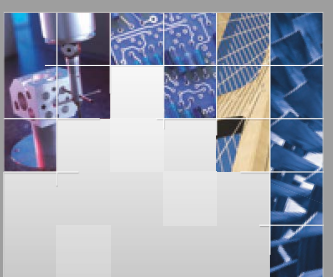

\section{Enfincering}
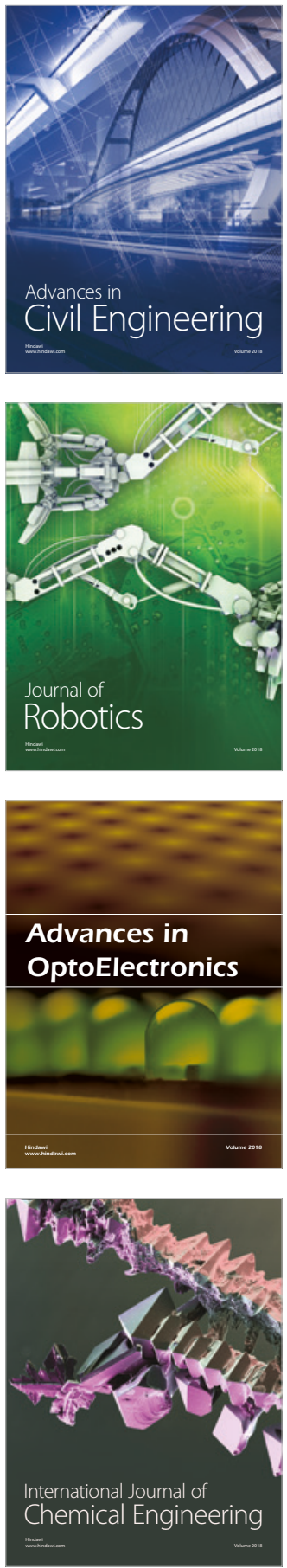

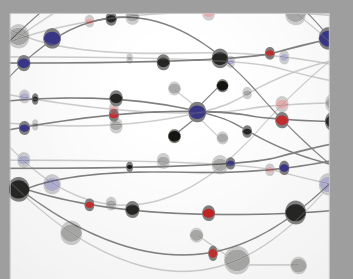

\section{Rotating \\ Machinery}

The Scientific World Journal

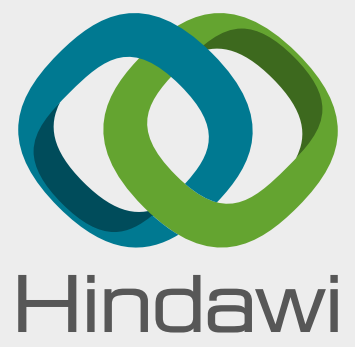

Submit your manuscripts at

www.hindawi.com
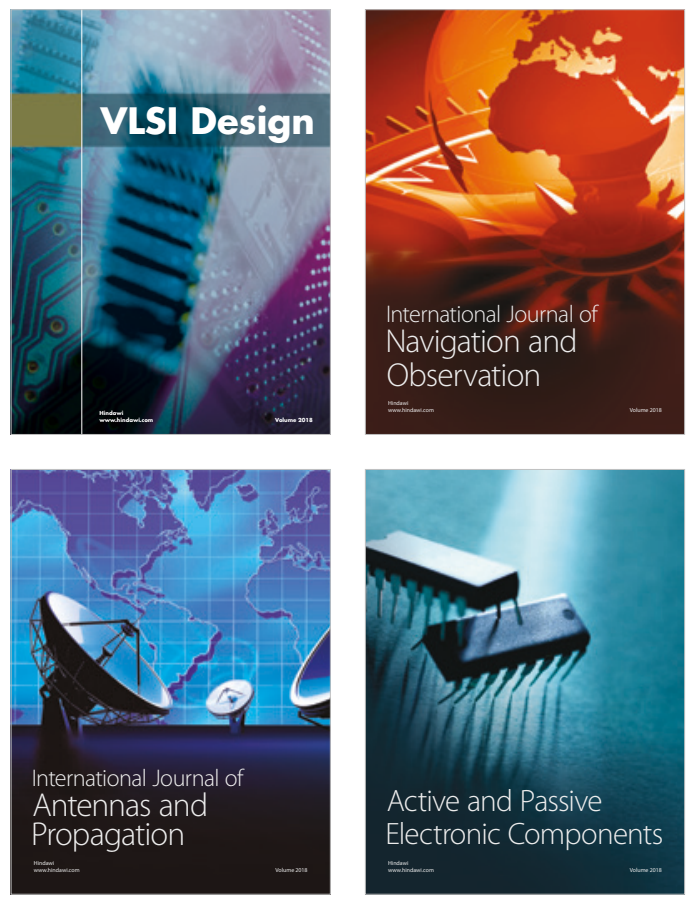
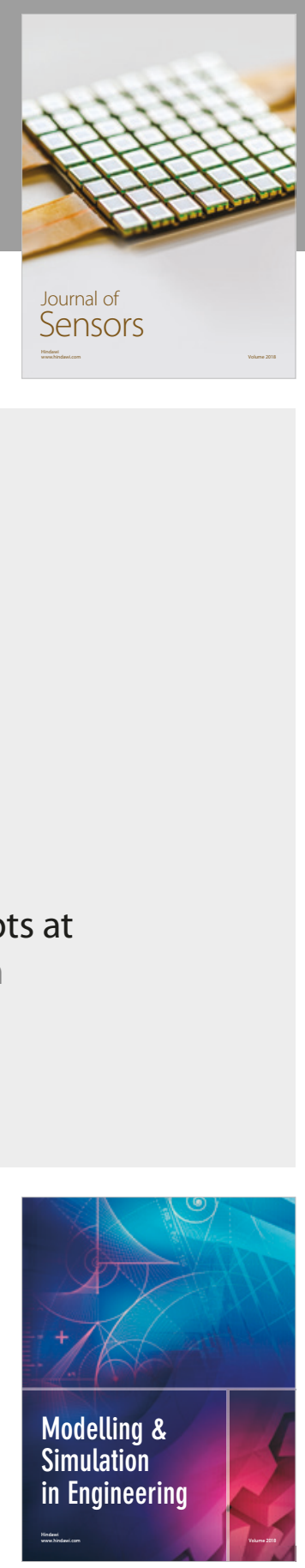

\section{Advances \\ Multimedia}
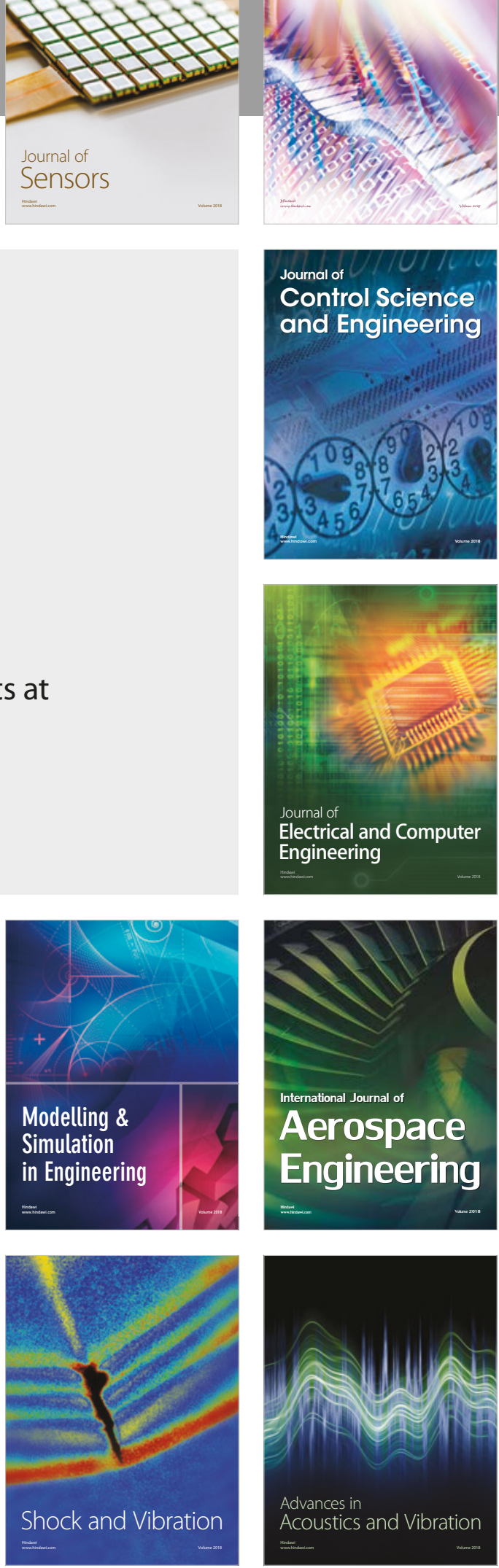